TRANSACTIONS OF THE

AMERICAN MATHEMATICAL SOCIETY

Volume 350, Number 10, October 1998, Pages 4065-4087

S 0002-9947(98)02292-2

\title{
SELF-SIMILAR MEASURES AND INTERSECTIONS OF CANTOR SETS
}

\author{
YUVAL PERES AND BORIS SOLOMYAK
}

\begin{abstract}
It is natural to expect that the arithmetic sum of two Cantor sets should have positive Lebesgue measure if the sum of their dimensions exceeds 1 , but there are many known counterexamples, e.g. when both sets are the middle- $\alpha$ Cantor set and $\alpha \in\left(\frac{1}{3}, \frac{1}{2}\right)$. We show that for any compact set $K$ and for a.e. $\alpha \in(0,1)$, the arithmetic sum of $K$ and the middle- $\alpha$ Cantor set does indeed have positive Lebesgue measure when the sum of their Hausdorff dimensions exceeds 1 . In this case we also determine the essential supremum, as the translation parameter $t$ varies, of the dimension of the intersection of $K+t$ with the middle- $\alpha$ Cantor set.

We also establish a new property of the infinite Bernoulli convolutions $\nu_{\lambda}^{p}$ (the distributions of random series $\sum_{n=0}^{\infty} \pm \lambda^{n}$, where the signs are chosen independently with probabilities $(p, 1-p)$ ). Let $1 \leq q_{1}<q_{2} \leq 2$. For $p \neq \frac{1}{2}$ near $\frac{1}{2}$ and for a.e. $\lambda$ in some nonempty interval, $\nu_{\lambda}^{p}$ is absolutely continuous and its density is in $L^{q_{1}}$ but not in $L^{q_{2}}$. We also answer a question of Kahane concerning the Fourier transform of $\nu_{\lambda}^{1 / 2}$.
\end{abstract}

\section{IntRoduCtion AND MAIN RESUlTS}

In this paper we consider one-parameter families of homogeneous Cantor sets, and determine the measure-theoretic properties of their sums and intersections for typical values of the parameter. We also obtain new results on the densities of infinite Bernoulli convolutions. In all previous works we know where absolute continuity of a parametrized family of measures for almost all parameters is established (e.g., Erdős [6], Kaufman [13], Mattila [18], Solomyak [25], Peres and Solomyak [23]) this is done by proving the existence of densities in $L^{2}$; a novel feature of the present work is that almost sure absolute continuity is established in cases where the densities are only in $L^{q}$ for certain $q<2$.

Let us first indicate our results for the special case of the sets

$$
\mathcal{K}_{\lambda}=\left\{(1-\lambda) \sum_{n=0}^{\infty} a_{n} \lambda^{n}: a_{n} \in\{0,1\}\right\} .
$$

(It is easily checked that $\mathcal{K}_{\lambda}$ is the middle- $\alpha$ Cantor set for $\alpha=1-2 \lambda$.) Let $K \subset \mathbf{R}$ be any compact set. We show that for a.e. $\lambda \in(0,1 / 2)$ such that the sum of Hausdorff dimensions $\operatorname{dim}_{H} K+\operatorname{dim}_{H} \mathcal{K}_{\lambda}$ is greater than 1:

1. the arithmetic sum $K+\mathcal{K}_{\lambda}$ has positive Lebesgue measure (see Theorem 1.1);

Received by the editors September 9, 1996.

1991 Mathematics Subject Classification. Primary 26A46; Secondary 26A30, 28A78, 28A80.

Key words and phrases. Cantor sets, Hausdorff dimension, self-similar measures.

The authors were supported in part by NSF grants DMS-9404391 and DMS-9500744.

(C)1998 American Mathematical Society 
2. if $K$ has positive Hausdorff measure in its dimension, then the equality

$$
\operatorname{dim}_{H}\left[(K+t) \cap \mathcal{K}_{\lambda}\right]=\operatorname{dim}_{H} K+\operatorname{dim}_{H} \mathcal{K}_{\lambda}-1
$$

holds for a set of parameters $t$ of positive Lebesgue measure (see Theorem $1.2)$.

The proofs of Theorems 1.1-1.2 rely on techniques developed by Mattila [19], Ch. 9-10, to prove projection and intersection theorems. Our situation is different since the dependence on the parameter $\lambda$ is nonlinear, but appropriate estimates of power series allow us to handle this. For the simplest application of this technique in a nonlinear setting, see Peres and Solomyak [23].

Next, to illustrate our results on self-similar measures, we consider the special case of the infinite Bernoulli convolutions $\nu_{\lambda}^{p}$. For each $\lambda, p \in(0,1)$, the measure $\nu_{\lambda}^{p}$ is defined as the distribution of the random series

$$
\sum_{n=0}^{\infty} \pm \lambda^{n},
$$

where the signs are chosen independently at random with probabilities $(p, 1-p)$. The symmetric case $p=1 / 2$ has received the most attention, ever since the work of Erdös [5], [6]. For $p \neq 1 / 2$ near $1 / 2$, Corollary 1.4 below reveals an interesting phenomenon: $\nu_{\lambda}^{p}$ is singular for all $\lambda<p^{p}(1-p)^{1-p}$, and is absolutely continuous for a.e. $\lambda>p^{p}(1-p)^{1-p}$; moreover, for any $q_{1}<q_{2}$ in $[1,2]$ there is an interval $I\left(q_{1}, q_{2}\right)$ such that for Lebesgue-a.e. $\lambda \in I\left(q_{1}, q_{2}\right)$, the density of $\nu_{\lambda}^{p}$ is in the space $L^{q_{1}}$ but not in $L^{q_{2}}$. See Theorems 1.3 and 4.1 for more general statements. These generalizations allow us to answer a question of Kahane [12] on the rate of decay of the Fourier transform of $\nu_{\lambda}^{1 / 2}$; see Corollary 1.6.

Background. Palis and Takens [22] and the references therein show that the structure of arithmetic sums of Cantor sets is relevant to natural questions in smooth dynamics. Palis and Takens asked about the structure of the sums $\mathcal{K}_{\gamma}+\mathcal{K}_{\lambda}$ and conjectured that "typically" they have either zero Lebesgue measure or non-empty interior. Solomyak [27] showed that for each $\gamma \in(0,1 / 2)$, the set $\mathcal{K}_{\gamma}+\mathcal{K}_{\lambda}$ has positive Lebesgue measure for a.e. $\lambda \in(0,1 / 2)$ such that $\operatorname{dim}_{H} \mathcal{K}_{\gamma}+\operatorname{dim}_{H} \mathcal{K}_{\lambda}>1$. Theorem 1.1 extends this to more general sums of Cantor sets, where one of the summands is arbitrary.

Questions about arithmetic sums and differences of Cantor sets can be expressed using intersections of their translates, since $F-K=\{t \in \mathbf{R}:(K+t) \cap F \neq \emptyset\}$. When the intersection $(K+t) \cap F$ is nonempty, it is natural to inquire about its dimension. A classical result of Marstrand [17] implies that for any two compact sets $K, F \subset \mathbf{R}$

$$
\operatorname{dim}_{H}((K+t) \cap F) \leq \operatorname{dim}_{H}(K \times F)-1 \text { for Lebesgue-a.e. } t \in \mathbf{R} .
$$

(See Theorem 8.1 in Falconer [7], or Mattila [19], 13.12.) If one of $K$ and $F$ is a middle- $\alpha$ Cantor set (and more generally, if its Hausdorff dimension equals its upper Minkowski dimension), then $\operatorname{dim}_{H}(K \times F)=\operatorname{dim}_{H}(K)+\operatorname{dim}_{H}(F)$; see Mattila [19], 8.10.

Without further assumptions on $K$ and $F$ the inequality (4) can be strict; indeed Mattila [19], 13.18, exhibits two compact sets $K, F \subset \mathbf{R}$ of Hausdorff dimension 1 such that $(K+t) \cap F$ is either empty or a single point for every $t \in \mathbf{R}$. Hawkes [10] showed that when $K, F$ are both the middle-third Cantor set, the left-hand 
side of (4) equals $\frac{\log 2}{3 \log 3}$ for a.e. $t \in[-1,1]$, so the inequality in (4) is strict for a.e. $t \in \mathbf{R}$; Hawkes' result was extended by Kenyon and Peres [16] to other Cantor sets defined via expansions in integer bases. Theorem 1.2 indicates that such a "dimension drop" (for a.e. translate) is exceptional when one of $K, F$ is fixed and the other is chosen from a one-parameter family of homogeneous Cantor sets.

Erdős [5], [6] studied the symmetric infinite Bernoulli convolutions $\nu_{\lambda}^{1 / 2}$ defined as the distribution of the random series (3) with $p=1 / 2$.

For $\lambda<1 / 2$ the measure $\nu_{\lambda}^{1 / 2}$ is supported on a Cantor set, but for $\lambda \in[1 / 2,1)$ its closed support is an interval. Extending a classical result of Erdős [6], Solomyak [25] proved that for a.e. $\lambda \in(1 / 2,1)$ the measure $\nu_{\lambda}^{1 / 2}$ is absolutely continuous and has a density in $L^{2}$ (see Peres and Solomyak [23] for a shorter proof). Here we study more general one-parameter families of measures, where the transition from singularity to having an $L^{2}$-density can be more gradual.

Statement of results. Consider the family of homogeneous self-similar sets in $\mathbf{R}$,

$$
\mathcal{C}_{\lambda}=\left\{\sum_{j=0}^{\infty} x_{j}(\lambda) \lambda^{j}: x_{j}(\lambda) \in D(\lambda)\right\}, \quad \text { for } \lambda \in(0,1),
$$

where $D(\lambda)=\left\{d_{1}(\lambda), \ldots, d_{m}(\lambda)\right\}$ is a set of digits depending on $\lambda$, with $d_{i}(\lambda) \in$ $C^{1}[0,1]$. "Self-similar" means that $\mathcal{C}_{\lambda}$ is a union of $m$ rescaled copies of itself, and "homogeneous" means that the similarity ratio $\lambda$ is the same for all copies. The Cantor set $\mathcal{K}_{\lambda}$ corresponds to $m=2$ and the digits $d_{1}(\lambda)=0$ and $d_{2}(\lambda)=1-\lambda$. Say that the strong separation condition holds for $\lambda \in(0,1)$ if

$$
\left[d_{i}(\lambda)+\lambda \mathcal{C}_{\lambda}\right] \cap\left[d_{j}(\lambda)+\lambda \mathcal{C}_{\lambda}\right]=\emptyset \text { for } i \neq j .
$$

An easily checked sufficient condition for $(5)$ is $\lambda(b+1)<1$, where

$$
b:=\sup \left\{\left|\frac{d_{i}(\lambda)-d_{j}(\lambda)}{d_{k}(\lambda)-d_{l}(\lambda)}\right|: \lambda \in[0,1], i, j, k, l \leq m, k \neq l\right\}<\infty .
$$

The strong separation condition implies $\operatorname{dim}_{H} \mathcal{C}_{\lambda}=\frac{\log m}{\log (1 / \lambda)}<1$, so $\lambda<m^{-1}$.

Theorem 1.1. Suppose that $K$ is a compact set on the real line and $J \subset(0,1)$ is an interval such that the family $\left\{\mathcal{C}_{\lambda}\right\}$ satisfies the strong separation condition (5) for all $\lambda \in J$. Then

(a) for a.e. $\lambda \in J$ such that $\operatorname{dim}_{H} K+\operatorname{dim}_{H} \mathcal{C}_{\lambda}<1$, we have

$$
\operatorname{dim}_{H}\left(K+\mathcal{C}_{\lambda}\right)=\operatorname{dim}_{H} K+\operatorname{dim}_{H} \mathcal{C}_{\lambda}
$$

(b) for a.e. $\lambda \in J$ such that $\operatorname{dim}_{H} K+\operatorname{dim}_{H} \mathcal{C}_{\lambda}>1$, the set $K+\mathcal{C}_{\lambda}$ has positive Lebesgue measure.

Let $\mathcal{L}$ denote Lebesgue measure on $\mathbf{R}$. In the case when $\operatorname{dim}_{H} K+\operatorname{dim}_{H} \mathcal{C}_{\lambda}>1$, Theorem 1.1(b) implies that for almost every $\lambda$ we have $\mathcal{L}\left(\mathcal{C}_{\lambda}-K\right)>0$. This means that there exists a set $A_{\lambda}$ with $\mathcal{L}\left(A_{\lambda}\right)>0$, such that $(K+t) \cap \mathcal{C}_{\lambda} \neq \emptyset$ for $t \in A_{\lambda}$. The next theorem gives much more accurate information about these intersections. Denote by $\mathcal{H}^{\alpha}(K)$ the $\alpha$-dimensional Hausdorff measure of the set $K$.

Theorem 1.2. Suppose $K \subset \mathbf{R}$ is compact with $\mathcal{H}^{\alpha}(K)>0$, and $J \subset(0,1)$ is an interval such that the family $\mathcal{C}_{\lambda}$ satisfies the strong separation condition (5) for all $\lambda \in J$. Then for a.e. $\lambda \in J$ such that $\alpha+\operatorname{dim}_{H} \mathcal{C}_{\lambda}>1$, there exists a set $A_{\lambda}$ of positive Lebesgue measure such that

$$
\operatorname{dim}_{H}\left[(K+t) \cap \mathcal{C}_{\lambda}\right] \geq \alpha+\operatorname{dim}_{H} \mathcal{C}_{\lambda}-1 \quad \text { for } t \in A_{\lambda} .
$$




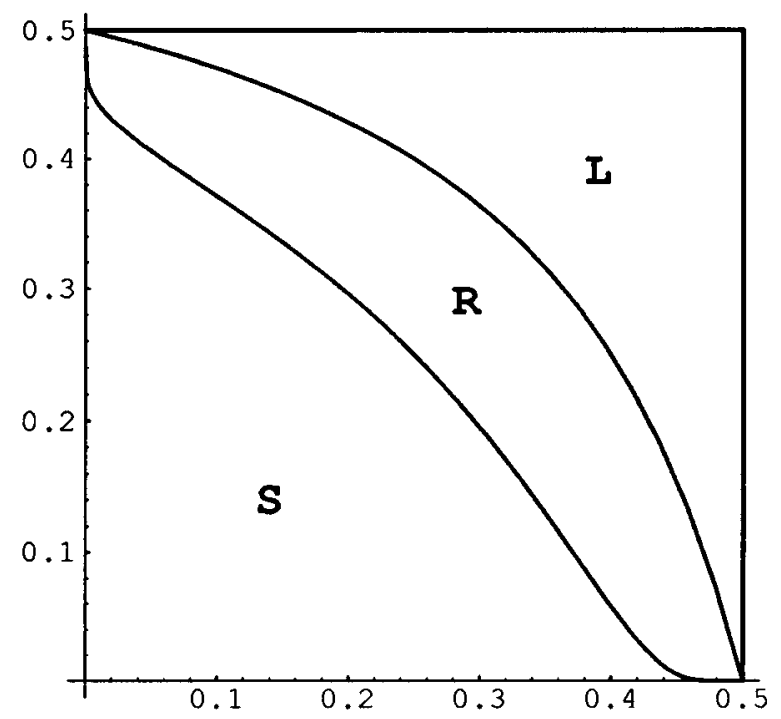

Figure 1. The $(\gamma, \lambda)$-plane: on the structure of $\mathcal{K}_{\gamma}+\mathcal{K}_{\lambda}$

If $\alpha=\operatorname{dim}_{H} K$ (which means that $K$ has positive Hausdorff measure in its dimension), then the inequality (7) becomes an equality for a.e. $t \in A_{\lambda}$.

Example: The Cantor sets $\mathcal{K}_{\lambda}$. The situation for the Cantor sets $\mathcal{K}_{\lambda}$ is illustrated in Figure 1. The region labelled $S$ is where $\frac{\log 2}{\log (1 / \gamma)}+\frac{\log 2}{\log (1 / \lambda)}=\operatorname{dim}_{H} \mathcal{K}_{\gamma}+$ $\operatorname{dim}_{H} \mathcal{K}_{\lambda}<1$, so $\operatorname{dim}_{H}\left(\mathcal{K}_{\gamma}+\mathcal{K}_{\lambda}\right)<1$. Theorem 1.1(a) says that in this region for a.e. point on each horizontal line, (and so by symmetry, every vertical line), $\operatorname{dim}_{H}\left(\mathcal{K}_{\gamma}+\mathcal{K}_{\lambda}\right)=\operatorname{dim}_{H} \mathcal{K}_{\gamma}+\operatorname{dim}_{H} \mathcal{K}_{\lambda}$. The region $L$ is where $\frac{\lambda}{1-2 \lambda} \frac{\gamma}{1-2 \gamma}>1$. Then by the Gap Lemma of Newhouse [21], the set $\mathcal{K}_{\gamma}+\mathcal{K}_{\lambda}$ is an interval. In the region $R$, Theorem 1.1(b) says that for a.e. point (on each horizontal and vertical line) the sum $\mathcal{K}_{\gamma}+\mathcal{K}_{\lambda}$ has positive Lebesgue measure. (This special case was originally obtained by Solomyak [27] using the Fourier transform, rather than the more direct method based on differentiation of measures employed here.)

Theorem 1.2 implies that for a.e. point $(\gamma, \lambda) \in R \cup L$, the set of translation parameters $t$ such that (2) holds with $K=\mathcal{K}_{\gamma}$ has positive Lebesgue measure.

The words "for almost every $\lambda$ " cannot be omitted in Theorems 1.1 and 1.2. Indeed, it is well-known that the sum $\mathcal{K}_{\lambda}+\mathcal{K}_{\lambda}$ is a Cantor set of Hausdorff dimension $\frac{\log 3}{\log (1 / \lambda)}$ for $\lambda<1 / 3$. Thus, on the diagonal $\gamma=\lambda \in(0,1 / 3)$, the Hausdorff dimension is lower than that given by Theorem 1.1. Some other exceptions were obtained by Keane and Smorodinsky [14]; see Solomyak [27].

We derive Theorem 1.1(b) from a criterion for absolute continuity of the convolution of a self-similar measure and an arbitrary measure satisfying a Frostman condition (Theorem 2.1 below). Self-similar measures have been studied extensively in their own right (see, e.g., Hutchinson [11] and Strichartz [28]). Given a probability vector $\mathbf{p}=\left(p_{1}, \ldots, p_{m}\right)$ and the digit set $D=\left\{d_{i}(\lambda)\right\}_{i=1}^{m}$, the self-similar measure $\nu_{\lambda}=\nu_{\lambda}(D, \mathbf{p})$ on $\mathbf{R}$ may be defined as the unique probability measure 
which satisfies

$$
\nu_{\lambda}=\sum_{i=1}^{m} p_{i}\left(\nu_{\lambda} \circ S_{i}^{-1}\right),
$$

where $S_{i}(x)=\lambda x+d_{i}(\lambda)$ (see Hutchinson [11]). Alternatively, consider the sequence space $\Omega=\{1, \ldots, m\} \mathbf{Z}_{+}$with the Bernoulli product measure $\mu=\mathbf{p}^{\mathbf{Z}_{+}}$on it, and the map $\Pi_{\lambda}: \Omega \rightarrow \mathbf{R}$ given by

$$
\Pi_{\lambda}(\omega)=\sum_{j=0}^{\infty} d_{\omega_{j}}(\lambda) \lambda^{j} .
$$

Then $\mathcal{C}_{\lambda}=\Pi_{\lambda}(\Omega)$ and

$$
\nu_{\lambda}(D, \mathbf{p})=\mu \circ \Pi_{\lambda}^{-1} .
$$

In the special case when $\mathcal{C}_{\lambda}=\mathcal{K}_{\lambda}$ and $\mu$ is Bernoulli $(1 / 2,1 / 2)$, this is the usual Cantor-Lebesgue measure.

Note that the strong separation condition (5) on an interval $J$ is equivalent to the property:

$$
\lambda \mapsto \Pi_{\lambda}(\omega)-\Pi_{\lambda}(\tau) \text { has no zeros on } J \text { for } \omega \neq \tau \text {. }
$$

Next we consider self-similar measures $\nu_{\lambda}$ without assuming strong separation. Say that the transversality condition holds on an interval $J \subset(0,1)$ if

$$
\lambda \mapsto \Pi_{\lambda}(\omega)-\Pi_{\lambda}(\tau) \text { has no double zeros on } J \text { for } \omega \neq \tau \text {. }
$$

Here a "double zero" for $f$ means $\lambda_{0}$ such that $f\left(\lambda_{0}\right)=f^{\prime}\left(\lambda_{0}\right)=0$ (so it includes zeros of higher order as well). An equivalent way to state the transversality condition is to say that the graphs of the functions $\lambda \mapsto \Pi_{\lambda}(\omega)$ and $\lambda \mapsto \Pi_{\lambda}(\tau)$ defined on $J$ intersect transversally (if at all).

If a measure $\nu$ is absolutely continuous (with respect to Lebesgue measure $\mathcal{L}$ ), then $\frac{d \nu_{\lambda}}{d x}$ will denote its Radon-Nikodým derivative, referred to as its density.

Theorem 1.3. Suppose that $J \subset(0,1)$ is an interval such that the family $\left\{\Pi_{\lambda}\right\}$ satisfies the transversality condition (11) on $J$.

(a) The self-similar measure $\nu_{\lambda}$ is absolutely continuous for a.e. $\lambda>\prod_{i=1}^{m} p_{i}^{p_{i}}$ such that $\lambda \in J$, and singular for all $\lambda<\prod_{i=1}^{m} p_{i}^{p_{i}}$.

(b) Let $q \in(1,2]$. Then for a.e. $\lambda>\left[p_{1}^{q}+\cdots+p_{m}^{q}\right]^{\frac{1}{q-1}}$ such that $\lambda \in J$, the measure $\nu_{\lambda}$ is absolutely continuous with a density in $L^{q}$.

(c) For any $q>1$ and all $\lambda \in(0,1)$, if $\nu_{\lambda}$ is absolutely continuous with respect to Lebesgue measure and its density $\frac{d \nu_{\lambda}}{d x}$ is in $L^{q}(\mathbf{R})$, then $\lambda \geq\left[p_{1}^{q}+\cdots+p_{m}^{q}\right]^{\frac{1}{q-1}}$.

In Section 5 we discuss how to verify transversality. An easily checked sufficient condition for transversality is $\lambda(\sqrt{b}+1)<1$, where $b$ is given by (6). This is useful only for $m>2$; the case $m=2$ is discussed below. The singularity assertion in part (a) is straightforward, and is included only for comparison. Observe that if $\nu_{\lambda}(D, \mathbf{p})$ is absolutely continuous with bounded density, then necessarily $\lambda \geq \max p_{i}$.

Example: The case of two digits. Let $m=2$. Then, up to an affine change of variable, we can assume that $D=\{ \pm 1\}$, so $\nu_{\lambda}(D, \mathbf{p})$ is the distribution of the random series $\sum_{n=0}^{\infty} \pm \lambda^{n}$, where the signs are chosen independently with probabilities $\mathbf{p}=(p, 1-p)$. In this case we denote $\nu_{\lambda}(D, \mathbf{p})$ simply by $\nu_{\lambda}^{p}$. We will see in Corollary 5.2 that the transversality condition holds for $\lambda \in[0,0.64]$. Thus, for 
$p \in[0.17,0.83]$ Theorem 1.3(a) implies absolute continuity of $\nu_{\lambda}^{p}$ for a.e. $\lambda$ in some interval. Sometimes one can go up to $\lambda=1$ using additional considerations. In Solomyak [25] this is done for $p=\frac{1}{2}$. Here we extend this to $p$ in a neighborhood of $1 / 2$ :

Corollary 1.4. Let $m=2$ and $p \in[1 / 3,2 / 3]$. Then $\nu_{\lambda}^{p}$ is absolutely continuous for a.e. $\lambda \in\left[p^{p}(1-p)^{1-p}, 1\right)$, and has $L^{q}$-density for a.e. $\lambda \in\left[\left(p^{q}+(1-p)^{q}\right)^{\frac{1}{q-1}}, 1\right)$, where $q \in(1,2]$.

Remarks. 1. The interval $[1 / 3,2 / 3]$ in this corollary can be enlarged with more work, but another new idea is needed to extend the corollary to all $p \in(0,1)$.

2. The measure $\nu_{\lambda}^{p}$ is known to be singular if $\lambda$ is a reciprocal of a PV-number (an algebraic integer whose conjugates lie inside the unit disk). This was proved by Erdős [5] for $p=1 / 2$, and his proof works for all $p \in(0,1)$.

Example: $\boldsymbol{D}=\{-\mathbf{1}, \mathbf{0}, \mathbf{1}\}$ and $\mathbf{p}=\left(\frac{1}{4}, \frac{1}{2}, \frac{1}{4}\right)$. Denote by $\eta_{\lambda}=\nu_{\lambda}(D, \mathbf{p})$ the corresponding self-similar measure. It is easy to see that $\eta_{\lambda}$ is the convolution square of the measure $\nu_{\lambda}^{1 / 2}$. The density of $\eta_{\lambda}$, when it exists, solves a functional equation known as the "Schilling equation", see e.g. Borwein and Girgensohn [3] and Baron, Simon and Volkmann [1]. Derfel and Schilling [4] noted that the result mentioned above, that $\nu_{\lambda}^{1 / 2}$ has a density in $L^{2}$ for a.e. $\lambda \in\left(\frac{1}{2}, 1\right)$, implies that the convolution square $\eta_{\lambda}$ has a continuous density for a.e. $\lambda \in\left(\frac{1}{2}, 1\right)$.

Corollary 1.5. The measure $\eta_{\lambda}$ is absolutely continuous for a.e. $\lambda \in\left(\frac{1}{2 \sqrt{2}}, 1\right)$ and singular for all $\lambda<\frac{1}{2 \sqrt{2}}$. For all $q>1$ and $\lambda<\lambda_{q}^{*}=\left(2^{-q}+2 \cdot 4^{-q}\right)^{\frac{1}{q-1}}$ the measure $\eta_{\lambda}$ cannot have a density in $L^{q}$, but $\eta_{\lambda}$ has an $L^{q}$-density for a.e. $\lambda \in\left[\lambda_{q}^{*}, 1\right)$ provided that $q \in(1,2]$.

The Fourier transform of $\nu_{\lambda}^{1 / 2}$ and a question of Kahane. Kahane [12] considered the Fourier transform $\widehat{\nu_{\lambda}^{1 / 2}}(u)=\prod_{n=0}^{\infty} \cos \left(\lambda^{n} u\right)$ of $\nu_{\lambda}^{1 / 2}$ and the function $g(\lambda)=\sup \left\{\beta \mid \widehat{\nu_{\lambda}^{1 / 2}}(u)=O\left(u^{-\beta}\right)\right\}$ which measures the rate of decay of this transform. In Question 3 (p. 121) of that paper, Kahane asked whether $g(\lambda)=\frac{-\log 2}{2 \log \lambda}$ for a.e. $\lambda \in(0,1)$. The following corollary implies a negative answer to Kahane's question, and yields some positive information as well. It shows that the generalizations considered in Theorem 1.3 are useful even if one only cares about $\nu_{\lambda}^{1 / 2}$.

Corollary 1.6. Let $n \geq 1$ and denote $\tilde{\lambda}_{n}=\left(\begin{array}{c}2 n \\ n\end{array}\right) 2^{-2 n}$. The Fourier transform $\widehat{\nu_{\lambda}^{1 / 2}}(\cdot)$ is in $L^{2 n}(\mathbf{R})$ for a.e. $\lambda \in\left(\tilde{\lambda}_{n}, 1\right)$ and is not in $L^{2 n}(\mathbf{R})$ for all $\lambda<\tilde{\lambda}_{n}$.

Taking $n=2$, we see that $\widehat{\nu_{\lambda}^{1 / 2}} \notin L^{4}$ for all $\lambda<3 / 8$, while the a.e. formula for $g(\lambda)$ suggested by Kahane would require $\widehat{\nu_{\lambda}^{1 / 2}}$ to be in $L^{4}$ for a.e. $\lambda>1 / 4$.

The rest of the paper is organized as follows. In Section 2 we derive Theorem 1.1 from a more general theorem on absolute continuity of convolutions. This convolution result is also used in the proof of Theorem 1.2 concerning intersections, given in Section 3. A generalization of Theorem 1.3 on densities of self-similar measures is established in Section 4, which can be read independently of Sections 2-3. In Section 5 we explain how to check the transversality condition (11), and use it to prove corollaries $1.4-1.6$. We conclude in Section 6 with some remarks and open problems. 


\section{Absolutely continuous convolutions And proof of Theorem 1.1}

Part (a) of Theorem 1.1 is easier to prove than part (b). Part (b) is proved by convolving a Frostman measure $\eta$ on $K$ with the self-similar measure $\nu_{\lambda}(D, \mathbf{p})$ on $\mathcal{C}_{\lambda}$, where $\mathbf{p}=(1 / m, \ldots, 1 / m)$.

This scheme leads naturally to a more general set-up: Let $\eta$ be a finite measure on the real line $\mathbf{R}$ with compact support such that for some $\alpha \in(0,1)$, the Frostman condition is satisfied:

$$
\eta\left[B_{r}(x)\right] \leq C r^{\alpha}, \quad \text { for } x \in \mathbf{R} \text { and } r>0 .
$$

Recall the map $\Pi_{\lambda}$ defined in (9). For $\omega$ and $\tau$ in $\Omega$ denote $|\omega \wedge \tau|=\min \left\{i: \omega_{i} \neq \tau_{i}\right\}$. Let $\mu$ be a probability measure on $\Omega$ such that for some $s \in(0,1]$,

$$
(\mu \times \mu)\{(\omega, \tau):|\omega \wedge \tau|=k\}<C m^{-k s},
$$

and consider the projected measure

$$
\nu_{\lambda}=\mu \circ \Pi_{\lambda}^{-1} .
$$

Theorem 2.1. Suppose that the strong separation condition (5) holds for all $\lambda$ in an open interval $J$, and that the conditions (12) and (13) hold. Then the measure $\eta * \nu_{\lambda}$ is absolutely continuous and has a density in $L^{2}$ for a.e. $\lambda \in J$ such that

$$
\alpha+\frac{s \log m}{\log (1 / \lambda)}>1
$$

Theorem 1.1(b) will follow by letting $\eta$ be a Frostman measure on $K$ (see Mattila [19], 8.8) and taking $\mu$ Bernoulli with $\mathbf{p}=(1 / m, \ldots, 1 / m)$, so that (13) holds with $s=1$.

We start by introducing some notation. For two sequences $\omega$ and $\tau$ in $\Omega$ let

$$
\phi_{\omega, \tau}(\lambda)=\Pi_{\lambda}(\omega)-\Pi_{\lambda}(\tau)=\sum_{j=|\omega \wedge \tau|}^{\infty}\left(d_{\omega_{j}}(\lambda)-d_{\tau_{j}}(\lambda)\right) \lambda^{j} .
$$

Let $I=\left[\lambda_{0}, \lambda_{1}\right] \subset J$ be a closed interval. Since the strong separation condition (5) holds for $\lambda \in I$, there exists $\delta_{I}>0$ such that

$$
\forall \omega, \tau \in \Omega, \quad\left(\omega_{0} \neq \tau_{0}\right) \Rightarrow\left|\phi_{\omega, \tau}(\lambda)\right| \geq \delta_{I} \quad \text { for } \quad \lambda \in I .
$$

Indeed, the absolute value in (16) represents the distance between a point in $d_{\omega_{0}}+$ $\lambda \mathcal{C}_{\lambda}$ and a point in $d_{\tau_{0}}+\lambda \mathcal{C}_{\lambda}$.

The proofs of Theorems 1.1, 1.2 and 2.1 rely on the derivative of $\phi_{\omega, \tau}(\lambda)$ being nonzero for all $\omega \neq \tau$ such that $|\omega \wedge \tau|$ is sufficiently large. Denote

$$
\begin{aligned}
G & =\max \left\{\left|d_{j}(\lambda)\right|: j \leq m, \lambda \in[0,1]\right\}, \\
G^{\prime} & =\max \left\{\left|d_{j}^{\prime}(\lambda)\right|: j \leq m, \lambda \in[0,1]\right\}
\end{aligned}
$$

and let

$$
N_{I}=\left\lfloor 4 \delta_{I}^{-1}\left(G \lambda_{1}\left(1-\lambda_{1}\right)^{-2}+G^{\prime} \lambda_{1}\left(1-\lambda_{1}\right)^{-1}\right)\right\rfloor+1,
$$

where $\delta_{I}$ comes from (16).

Lemma 2.2. For $\phi=\phi_{\omega, \tau}$ and $k=|\omega \wedge \tau| \geq N_{I}$ we have

$$
\left|\phi^{\prime}(\lambda)\right| \geq(1 / 2) k \delta_{I} \lambda^{k-1} \quad \text { for } \lambda \in I \text {. }
$$


Proof. Let $\phi(\lambda)=\sum_{j=k}^{\infty} \phi_{j}(\lambda) \lambda^{j}$. We have

$$
\begin{aligned}
\left|\phi^{\prime}(\lambda)\right| & =\left|k \sum_{j=k}^{\infty} \phi_{j}(\lambda) \lambda^{j-1}+\sum_{j=k+1}^{\infty}(j-k) \phi_{j}(\lambda) \lambda^{j-1}+\sum_{j=k}^{\infty} \phi_{j}^{\prime}(\lambda) \lambda^{j}\right| \\
& \geq k \lambda^{k-1}\left|\sum_{j=0}^{\infty} \phi_{j+k}(\lambda) \lambda^{j}\right|-\lambda^{k-1}\left|\sum_{j=1}^{\infty} j \phi_{j+k}(\lambda) \lambda^{j}\right|-\lambda^{k}\left|\sum_{j=0}^{\infty} \phi_{j+k}^{\prime}(\lambda) \lambda^{j}\right| .
\end{aligned}
$$

Since $\phi_{k}(\lambda) \neq 0$, it follows from (16) that $\left|\sum_{j=0}^{\infty} \phi_{j+k}(\lambda) \lambda^{j}\right| \geq \delta_{I}$. Together with the inequalities $\left|\phi_{j}(\lambda)\right| \leq 2 G$ and $\left|\phi_{j}^{\prime}(\lambda)\right| \leq 2 G^{\prime}$, this implies for $\lambda \in I=\left[\lambda_{0}, \lambda_{1}\right]$ that

$$
\begin{aligned}
\left|\phi^{\prime}(\lambda)\right| & \geq k \lambda^{k-1} \delta_{I}-\lambda^{k-1} 2 G \frac{\lambda}{(1-\lambda)^{2}}-\lambda^{k} 2 G^{\prime} \frac{1}{1-\lambda} \\
& \geq \lambda^{k-1}\left[k \delta_{I}-\frac{2 G \lambda_{1}}{\left(1-\lambda_{1}\right)^{2}}-\frac{2 G^{\prime} \lambda_{1}}{\left(1-\lambda_{1}\right)}\right] \\
& \geq(1 / 2) k \delta_{I} \lambda^{k-1},
\end{aligned}
$$

since $\frac{2 G \lambda_{1}}{\left(1-\lambda_{1}\right)^{2}}+\frac{2 G^{\prime} \lambda_{1}}{\left(1-\lambda_{1}\right)} \leq N_{I} \delta_{I} / 2 \leq k \delta_{I} / 2$ by $(17)$.

Lemma 2.3. Let $I=\left[\lambda_{0}, \lambda_{1}\right] \subset J$ and assume that $k=|\omega \wedge \tau| \geq N_{I}$, where $N_{I}$ is given by (17). Then:

(a) There exists $C_{1}$ such that for all $y, z \in \mathbf{R}$,

$$
\mathcal{L}\left\{\lambda \in I:\left|y-z+\phi_{\omega, \tau}(\lambda)\right| \leq r\right\}<C_{1} \min \left\{1, r \lambda_{0}^{-k}\right\} .
$$

(b) There exists $C_{2}$ such that

$$
\left|\phi_{\omega, \tau}(\lambda)\right| \leq \frac{C_{2}}{2} \lambda_{1}^{k} \quad \text { for } \lambda \in I=\left[\lambda_{0}, \lambda_{1}\right] .
$$

(c) For any $\beta \in(0,1)$ there exists $C_{3}=C_{3}(\beta)$ such that

$$
\int_{I}\left|y-z+\phi_{\omega, \tau}(\lambda)\right|^{-\beta} d \lambda \leq \begin{cases}C_{3} \lambda_{0}^{-k \beta} & \text { for all } y, z \\ 2^{\beta}|y-z|^{-\beta} & \text { if }|y-z|>C_{2} \lambda_{1}^{k} .\end{cases}
$$

Proof. (a) Let $\phi(\lambda)=\phi_{\omega, \tau}(\lambda)$ and consider $\psi(\lambda)=y-z+\phi(\lambda)$. We need to estimate the length of $\Lambda_{r}=\{\lambda \in I:|\psi(\lambda)| \leq r\}$. By Lemma 2.2, $\left|\psi^{\prime}(\lambda)\right|=$ $\left|\phi^{\prime}(\lambda)\right| \geq(1 / 2) k \delta_{I} \lambda_{0}^{k-1}$ for $\lambda \in I$, so $\Lambda_{r}$ is at most a single interval by monotonicity of $\psi$, and

$$
\mathcal{L}\left(\Lambda_{r}\right) \leq \frac{2 r}{(1 / 2) k \delta_{I} \lambda_{0}^{k-1}}<\frac{4 r}{\delta_{I} \lambda_{0}^{k}} .
$$

The inequality $\mathcal{L}\left(\Lambda_{r}\right) \leq 1$ is obvious.

(b) Using the definition of $G$ and summing a geometric series gives $\left|\phi_{\omega, \tau}(\lambda)\right| \leq$ $2 G \lambda^{k}(1-\lambda)^{-1} \leq 2 G \lambda_{1}^{k}\left(1-\lambda_{1}\right)^{-1}$ for $\lambda \in I$, so setting $C_{2}=4 G\left(1-\lambda_{1}\right)^{-1}$ yields (18).

(c) Since $\beta<1$, the first inequality is a consequence of part (a), where the distribution function of the integrand was bounded. The second inequality follows from (b). 
Proof of Theorem 1.1(a). The estimate $\operatorname{dim}_{H}\left(K+\mathcal{C}_{\lambda}\right) \leq \operatorname{dim}_{H} K+\operatorname{dim}_{H} \mathcal{C}_{\lambda}$ is wellknown, so we only need to get the lower bound. Recall that $\operatorname{dim}_{H} \mathcal{C}_{\lambda}=\frac{\log m}{\log (1 / \lambda)}$. Fix an arbitrary $\alpha_{*}<\operatorname{dim}_{H} K$ and let $\alpha \in\left(\alpha_{*}, \operatorname{dim}_{H} K\right)$. Suppose that $\lambda_{0} \in J$ satisfies $\alpha_{*}+\frac{\log m}{\log \left(1 / \lambda_{0}\right)}<1$. Then we can find $\lambda_{1}>\lambda_{0}$ so that $I=\left[\lambda_{0}, \lambda_{1}\right] \subset J$,

$$
\beta=\alpha_{*}+\frac{\log m}{\log \left(1 / \lambda_{1}\right)}<1
$$

and

$$
\beta \log \left(1 / \lambda_{0}\right)-\alpha \log \left(1 / \lambda_{1}\right)<\log m .
$$

Since $\alpha<\operatorname{dim}_{H} K$, there is a measure $\eta$ on $K$ satisfying the Frostman condition (12), see e.g. Mattila [19], Ch.8. Let $\mu$ be the Bernoulli measure on $\Omega$ which assigns equal probability $1 / m$ to each symbol. To apply the preceding lemmas, we need to restrict the measure $\mu$ to a fixed cylinder set $W$ of the order $N_{I}$ given by (17). Consider the measure $\sigma_{\lambda}$ given by

$$
\sigma_{\lambda}=\left.\mu\right|_{W} \circ \Pi_{\lambda}^{-1} .
$$

The convolution $\eta * \sigma_{\lambda}$ is supported on $K+\Pi_{\lambda}(W) \subset K+\mathcal{C}_{\lambda}$, and we want to show that

$$
\mathcal{J}:=\int_{I} \int_{\mathbf{R}} \int_{\mathbf{R}}|x-v|^{-\beta} d\left(\eta * \sigma_{\lambda}\right)(x) d\left(\eta * \sigma_{\lambda}\right)(v) d \lambda<\infty .
$$

Using the definition of convolution and then making a change of variable, we get

$$
\begin{aligned}
\mathcal{J} & =\int_{I} \int_{K} \int_{\mathcal{C}_{\lambda}} \int_{K} \int_{\mathcal{C}_{\lambda}}\left|\left(y+y^{\prime}\right)-\left(z+z^{\prime}\right)\right|^{-\beta} d \sigma_{\lambda}\left(y^{\prime}\right) d \eta(y) d \sigma_{\lambda}\left(z^{\prime}\right) d \eta(z) d \lambda \\
& =\int_{I} \int_{K} \int_{W} \int_{K} \int_{W}\left|\left(y+\Pi_{\lambda}(\omega)\right)-\left(z+\Pi_{\lambda}(\tau)\right)\right|^{-\beta} d \mu(\omega) d \eta(y) d \mu(\tau) d \eta(z) d \lambda .
\end{aligned}
$$

By Fubini's Theorem and (15),

$$
\mathcal{J}=\iint_{K^{2}} \iint_{W^{2}} \int_{I}\left|(y-z)+\phi_{\omega, \tau}(\lambda)\right|^{-\beta} d \lambda d \mu(\omega) d \mu(\tau) d \eta(y) d \eta(z) .
$$

Since $(\mu \times \mu)\{(\omega, \tau):|t \wedge u|=k\}<m^{-k}$, the energy bounds in Lemma 2.3(c) yield

$$
\begin{aligned}
\mathcal{J} & \leq \tilde{C} \iint_{K^{2}}\left[\sum_{k:|y-z| \leq C_{2} \lambda_{1}^{k}} \lambda_{0}^{-\beta k} m^{-k}+\sum_{k:|y-z|>C_{2} \lambda_{1}^{k}}|y-z|^{-\beta} m^{-k}\right] d \eta(y) d \eta(z) \\
& =: \tilde{C}\left(\mathcal{I}_{1}+\mathcal{I}_{2}\right) .
\end{aligned}
$$

First we estimate $\mathcal{I}_{1}$. By (12),

$$
\mathcal{I}_{1}=\sum_{k \geq 0} \lambda_{0}^{-\beta k} m^{-k}(\eta \times \eta)\left\{(y, z):|y-z| \leq C_{2} \lambda_{1}^{k}\right\} \leq C \sum_{k \geq 0} \lambda_{0}^{-\beta k} m^{-k} \lambda_{1}^{k \alpha} .
$$

Condition (20) implies $\lambda_{0}^{-\beta} m^{-1} \lambda_{1}^{\alpha}<1$, so $\mathcal{I}_{1}<\infty$. Next, setting $\kappa(y, z)=$ $\frac{\log \left(C_{2}^{-1}|y-z|\right)}{\log \lambda_{1}}$, we get

$$
\mathcal{I}_{2} \leq C_{*} \iint_{K^{2}}|y-z|^{-\beta} m^{-\kappa(y, z)} d \eta(y) d \eta(z)
$$


and

$$
|y-z|^{-\beta} m^{-\kappa(y, z)}=C^{\prime}|y-z|^{-\beta}|y-z|^{\frac{\log m}{\log \left(1 / \lambda_{1}\right)}}=C^{\prime}|y-z|^{-\alpha_{*}} .
$$

Now, since $\alpha_{*}<\alpha$, condition (12) implies

$$
\iint_{K^{2}}|y-z|^{-\alpha_{*}} d \eta(y) d \eta(z)<\infty
$$

see e.g. Mattila [19], Ch.8. Thus $\mathcal{I}_{2}<\infty$ and (21) is established. Therefore $\eta * \sigma_{\lambda}$ has finite $\beta$-energy for Lebesgue-a.e. $\lambda \in I$; hence for all such $\lambda$ we have

$$
\operatorname{dim}_{H}\left(K+\mathcal{C}_{\lambda}\right) \geq \beta \geq \alpha_{*}+\frac{\log m}{\log (1 / \lambda)}
$$

see e.g. Mattila [19], Ch.8. Letting $\alpha_{*}$ tend to $\operatorname{dim}_{H} K$ completes the proof of Theorem 1.1(a).

Proof of Theorem 2.1 (which implies Theorem 1.1(b)). We are given a finite measure $\eta$ on $\mathbf{R}$ satisfying the Frostman condition (12). The measure $\mu$ on $\Omega$ is arbitrary satisfying (13), and $\nu_{\lambda}=\mu \circ \Pi_{\lambda}^{-1}$. Let $\lambda_{0} \in J$ be such that $\alpha+\frac{s \log m}{\log \left(1 / \lambda_{0}\right)}>1$. One can find $\lambda_{1}>\lambda_{0}$ so that (16) holds on $I=\left[\lambda_{0}, \lambda_{1}\right]$, and

$$
\alpha>\frac{\log \left(\lambda_{0} m^{s}\right)}{\log \lambda_{1}} \text {. }
$$

It suffices to prove that the convolution $\eta * \nu_{\lambda}$ has a density in $L^{2}$ for a.e. $\lambda \in I=$ $\left[\lambda_{0}, \lambda_{1}\right]$.

As in the proof of Theorem 1.1(a), we restrict $\mu$ to a cylinder set $W$ of order $N_{I}$, where $N_{I}$ is given by (17), and let $\sigma_{\lambda}=\left.\mu\right|_{W} \circ \Pi_{\lambda}^{-1}$. Since $\nu_{\lambda}$ is a linear combination of $m^{N_{I}}$ translated copies of $\sigma_{\lambda}$, if $\eta * \sigma_{\lambda}$ has a density in $L^{2}$ then so does $\eta * \nu_{\lambda}$. Consider the lower density of $\eta * \sigma_{\lambda}$,

$$
\underline{D}\left(\eta * \sigma_{\lambda}, x\right)=\liminf _{r \downarrow 0}(2 r)^{-1}\left(\eta * \sigma_{\lambda}\right)\left[B_{r}(x)\right],
$$

where $B_{r}(x)=[x-r, x+r]$. As in Mattila [19], 9.7, if

$$
\mathcal{J}_{\lambda}:=\int_{\mathbf{R}} \underline{D}\left(\eta * \sigma_{\lambda}, x\right) d\left(\eta * \sigma_{\lambda}\right)(x)<\infty,
$$

then $\underline{D}\left(\eta * \sigma_{\lambda}, x\right)$ is finite for $\left(\eta * \sigma_{\lambda}\right)$-a.e. $x$, and $\eta * \sigma_{\lambda}$ has a density in $L^{2}$. Thus, it is enough to show that

$$
\mathcal{S}:=\int_{I} \mathcal{J}_{\lambda} d \lambda<\infty
$$

By Fatou's Lemma,

$$
\mathcal{S} \leq \mathcal{S}_{1}:=\liminf _{r \downarrow 0}(2 r)^{-1} \int_{I} \int_{\mathbf{R}}\left(\eta * \sigma_{\lambda}\right)\left[B_{r}(x)\right] d\left(\eta * \sigma_{\lambda}\right)(x) d \lambda .
$$

Using the definition of convolution and making a change of variable, we obtain

$$
\mathcal{S}_{1}=\liminf _{r \downarrow 0}(2 r)^{-1} \int_{I} \int_{\mathbf{R}} \int_{W}\left(\eta * \sigma_{\lambda}\right)\left[B_{r}\left(y+\Pi_{\lambda}(\omega)\right)\right] d \mu(\omega) d \eta(y) d \lambda .
$$


Next, denoting by $\mathbf{1}_{A}$ the indicator function of a set $A$,

$$
\begin{array}{r}
\left(\eta * \sigma_{\lambda}\right)\left[B_{r}\left(y+\Pi_{\lambda}(\omega)\right)\right]=\int_{\mathbf{R}} \mathbf{1}_{B_{r}\left(y+\Pi_{\lambda}(\omega)\right)}(w) d\left(\eta * \sigma_{\lambda}\right)(w) \\
\quad=\int_{\mathbf{R}} \int_{W} \mathbf{1}_{\left\{(z, \tau): z+\Pi_{\lambda}(\tau) \in B_{r}\left(y+\Pi_{\lambda}(\omega)\right)\right\}}(z, \tau) d \mu(\tau) d \eta(z),
\end{array}
$$

Substituting this into (24) and reversing the order of integration yields

$$
\mathcal{S}_{1}=\liminf _{r \downarrow 0}(2 r)^{-1} \int_{\mathbf{R}} \int_{W} \int_{\mathbf{R}} \int_{W} \mathcal{L}\left(\Lambda_{r}(y, z, \omega, \tau)\right) d \mu(\tau) d \eta(z) d \mu(\omega) d \eta(y),
$$

where

$$
\begin{gathered}
\Lambda_{r}(y, z, \omega, \tau):=\left\{\lambda \in I:\left|\left(y+\Pi_{\lambda}(\omega)\right)-\left(z+\Pi_{\lambda}(\tau)\right)\right| \leq r\right\} \\
=\left\{\lambda \in I:\left|y-z+\phi_{\omega, \tau}(\lambda)\right| \leq r\right\} .
\end{gathered}
$$

Split the integral in (25) according to the distance between $y$ and $z$ :

$$
\begin{aligned}
& \int_{\mathbf{R}} \int_{W} \int_{\mathbf{R}} \int_{W} \mathcal{L}\left(\Lambda_{r}(y, z, \omega, \tau)\right) \\
& \quad=\iint_{\{|y-z|<2 r\}} \iint_{W^{2}}+\iint_{\{|y-z| \geq 2 r\}} \iint_{W^{2}}:=\mathcal{I}_{1}+\mathcal{I}_{2} .
\end{aligned}
$$

To complete the proof, it is sufficient to show that $\mathcal{I}_{1} \leq C r$ and $\mathcal{I}_{2} \leq C r$. By Lemma 2.3(a), (12) and (13),

$$
\mathcal{I}_{1} \leq C(2 r)^{\alpha} \sum_{k=N_{I}}^{\infty} \min \left\{1, r \lambda_{0}^{-k}\right\} m^{-k s} .
$$

Therefore, setting $k_{r}=\frac{\log r}{\log \lambda_{0}}$, we get

$$
\mathcal{I}_{1} \leq C(2 r)^{\alpha}\left[\sum_{k \leq k_{r}} r \lambda_{0}^{-k} m^{-k s}+\sum_{k>k_{r}} m^{-k s}\right] .
$$

Recall that the strong separation condition (5) implies $\lambda_{0}<1 / m$, so $\lambda_{0}^{-1} m^{-s}>1$. Summing the geometric series and using $\lambda_{0}^{k_{r}}=r$, we obtain

$$
\mathcal{I}_{1} \leq C r^{\alpha} m^{-k_{r} s} \text {. }
$$

Since $\alpha-\frac{\log m}{\log r} s k_{r}=\alpha+\frac{s \log m}{\log \left(1 / \lambda_{0}\right)}>1$, for $r<1$ we have $r^{\alpha} m^{-k_{r} s}<r$, and (28) implies $\mathcal{I}_{1} \leq C r$.

It remains to estimate $\mathcal{I}_{2}$, the second integral in (27). If $C_{2} \lambda_{1}^{k}<|y-z|$, then by (18), $\left|\phi_{\omega, \tau}(\lambda)\right|<|y-z| / 2$, and $\left|y-z+\phi_{\omega, \tau}(\lambda)\right|>|y-z| / 2$. When $|y-z| \geq 2 r$ this implies that the set $\Lambda_{r}(y, z, \omega, \tau)$ is empty. Denote $\kappa(y, z)=\frac{\log \left(C_{2}^{-1}|y-z|\right)}{\log \lambda_{1}}$. We 
obtain from Lemma 2.3(a) and (13), keeping in mind that $\lambda_{0} m^{s}<1$ :

$$
\begin{aligned}
\mathcal{I}_{2} & \leq \iint_{\mathbf{R}^{2}} \sum_{k \leq \kappa(y, z)} r \lambda_{0}^{-k} m^{-k s} d \eta(y) d \eta(z) \\
& \leq C r \iint_{\mathbf{R}^{2}}\left(\lambda_{0} m^{s}\right)^{-\kappa(y, z)} d \eta(y) d \eta(z) \\
& =C^{\prime} r \iint_{\mathbf{R}^{2}}|y-z|^{-\frac{\log \left(\lambda_{0} m^{s}\right)}{\log \lambda_{1}}} d \eta(y) d \eta(z) .
\end{aligned}
$$

But $\frac{\log \left(\lambda_{0} m^{s}\right)}{\log \lambda_{1}}<\alpha$ by (23), so $\mathcal{I}_{2}<C^{\prime \prime} r$ by (12), and the proof of Theorem 2.1 is complete.

\section{IntERSECTING TRANSLATES}

Proof of Theorem 1.2. Since $\mathcal{H}^{\alpha}(K)>0$, there is a Frostman measure $\eta$ on $K$ satisfying (12). Let $\tilde{\eta}(E)=\eta(-E)$. As in the proof of Theorem 1.1(a), $\mu$ is now the Bernoulli measure on $\Omega$ which assigns equal probability $1 / m$ to each symbol. Let $\nu_{\lambda}=\mu \circ \Pi_{\lambda}^{-1}$. Then (13) holds with $s=1$, so by Theorem 2.1, $\tilde{\eta} * \nu_{\lambda}$ is absolutely continuous with respect to $\mathcal{L}$ for a.e.

$$
\lambda \in J^{\prime}:=\left\{\lambda \in J: \alpha+\frac{\log m}{\log (1 / \lambda)}>1\right\} .
$$

We will show that for Lebesgue-a.e. $\lambda \in J^{\prime}$ and $\left(\tilde{\eta} * \nu_{\lambda}\right)$-a.e. $t \in \mathbf{R}$,

$$
\operatorname{dim}_{H}\left[(K+t) \cap \mathcal{C}_{\lambda}\right] \geq \alpha+\frac{\log m}{\log (1 / \lambda)}-1 .
$$

Define the set $A_{\lambda}$ to be the set of $t$ such that (29) holds. For Lebesgue-a.e. $\lambda \in J^{\prime}$ this set must have positive Lebesgue measure if it has full $\left(\tilde{\eta} * \nu_{\lambda}\right)$-measure; indeed it will follow that $A_{\lambda}$ contains Lebesgue a.e. $t$, where the density of $\tilde{\eta} * \nu_{\lambda}$ is positive. This will yield the statement of Theorem 1.2. If the set of "bad" $\lambda \in J^{\prime}$ (where $A_{\lambda}$ does not have full $\left(\tilde{\eta} * \nu_{\lambda}\right)$-measure) had positive Lebesgue measure, it would have a point of density; see Mattila [19], 2.14. To rule that out it is enough to prove that for any $\lambda_{0} \in J^{\prime}$ and any $\gamma$ such that

$$
1<\gamma<\gamma^{*}:=\alpha+\frac{\log m}{\log \left(1 / \lambda_{0}\right)},
$$

there exists $\lambda_{1}>\lambda_{0}$ such that

$$
\operatorname{dim}_{H}\left[(K+t) \cap \mathcal{C}_{\lambda}\right] \geq \gamma-1 \quad \text { for } \quad \mathcal{L} \text {-a.e. } \lambda \in I=\left[\lambda_{0}, \lambda_{1}\right] \text { and }\left(\tilde{\eta} * \nu_{\lambda}\right) \text {-a.e. } t \text {. }
$$

Fix $\gamma \in\left(1, \gamma^{*}\right)$. One can choose $\lambda_{1}>\lambda_{0}$ so that (16) holds and

$$
\alpha-\frac{\log \left(\lambda_{0} m\right)}{\log \lambda_{1}}>\gamma-1 \text {. }
$$

Denote

$$
l_{t}=\left\{\left(x, x^{\prime}\right) \in \mathbf{R}^{2}: x^{\prime}=x+t\right\} .
$$

As in Mattila [19], 13.12, we have

$$
\operatorname{dim}_{H}\left[(K+t) \cap \mathcal{C}_{\lambda}\right]=\operatorname{dim}_{H}\left[\left(K \times \mathcal{C}_{\lambda}\right) \cap l_{t}\right] .
$$


The plan is to show that for $\mathcal{L}$-a.e. $\lambda \in I$, the conditional measure of $\eta \times \nu_{\lambda}$ on $\left(K \times \mathcal{C}_{\lambda}\right) \cap l_{t}$ has finite $(\gamma-1)$-energy for $\left(\tilde{\eta} * \nu_{\lambda}\right)$-a.e. $t$. This will imply (30). In fact, as in Theorem 1.1 we will work with a restricted measure $\sigma_{\lambda}=\left.\mu\right|_{W} \circ \Pi_{\lambda}^{-1}$, where $W$ is a cylinder of order $N_{I}$ given by (17). Thus (30) will be proved for $\left(\tilde{\eta} * \sigma_{\lambda}\right)$-a.e. $t \in \mathbf{R}$. However, since $W$ can be any cylinder and since $\nu_{\lambda}$ is the sum of measures $\sigma_{\lambda}$ corresponding to all cylinders of order $N_{I}$, the full strength of (30) will follow.

The first step is to define the conditional measure. This can be done as in Mattila [19], Ch.10: for $\mathcal{L}$-a.e. $t \in \mathbf{R}$ there exists a non-negative Radon measure $\theta_{t, \lambda}$ on $\left(K \times \mathcal{C}_{\lambda}\right) \cap l_{t}$, such that for all $g \in C_{0}^{+}\left(\mathbf{R}^{2}\right)$,

$$
\int_{l_{t}} g\left(y, y^{\prime}\right) d \theta_{t, \lambda}\left(y, y^{\prime}\right)=\lim _{r \downarrow 0}(2 r)^{-1} \iint_{\left\{\left(y, y^{\prime}\right):\left|y^{\prime}-y-t\right| \leq r\right\}} g\left(y, y^{\prime}\right) d \eta(y) d \sigma_{\lambda}\left(y^{\prime}\right) .
$$

We are going to show that

$$
\mathcal{J}:=\int_{I} \int_{\mathbf{R}} \int_{l_{t}} \int_{l_{t}}\left\|\left(y, y^{\prime}\right)-\left(z, z^{\prime}\right)\right\|^{1-\gamma} d \theta_{t, \lambda}\left(y, y^{\prime}\right) d \theta_{t, \lambda}\left(z, z^{\prime}\right) d t d \lambda<\infty,
$$

where

$$
\|\left(y, y^{\prime}\right)-\left(z, z^{\prime}\right)||=\left|y-y^{\prime}\right|+\left|z-z^{\prime}\right| .
$$

Inequality (33) will imply that for a.e. $\lambda \in I$ and a.e. $t \in \mathbf{R}$, the measure $\theta_{t, \lambda}$ is either zero or positive with finite $(\gamma-1)$-energy. Now recall that by Theorem 2.1 the measure $\tilde{\eta} * \sigma_{\lambda}$ is absolutely continuous for a.e. $\lambda \in I$. For such $\lambda$, by the definition of convolution we have for $\mathcal{L}$-a.e. $t \in \mathbf{R}$

$$
\begin{aligned}
\frac{d\left(\tilde{\eta} * \sigma_{\lambda}\right)}{d x}(t) & =\lim _{r \downarrow 0}(2 r)^{-1} \iint_{\left\{\left(y, y^{\prime}\right):\left|y^{\prime}+y-t\right| \leq r\right\}} d \tilde{\eta}(y) d \sigma_{\lambda}\left(y^{\prime}\right) \\
& =\lim _{r \downarrow 0}(2 r)^{-1} \iint_{\left\{\left(y, y^{\prime}\right):\left|y^{\prime}-y-t\right| \leq r\right\}} d \eta(y) d \sigma_{\lambda}\left(y^{\prime}\right)=\theta_{t, \lambda}\left(l_{t}\right),
\end{aligned}
$$

using (32). Thus, $\theta_{t, \lambda}$ is positive for $\left(\tilde{\eta} * \sigma_{\lambda}\right)$-a.e. $t \in \mathbf{R}$, as desired.

It remains to establish (33). Any lower semicontinuous function $g: \mathbf{R}^{2} \rightarrow[0, \infty)$ is an increasing limit of a sequence of continuous functions, so we get from (32) that

$$
\int_{l_{t}} g\left(y, y^{\prime}\right) d \theta_{t, \lambda}\left(y, y^{\prime}\right) \leq \liminf _{r \downarrow 0}(2 r)^{-1} \iint_{\left\{\left(y, y^{\prime}\right):\left|y^{\prime}-y-t\right| \leq r\right\}} g\left(y, y^{\prime}\right) d \eta(y) d \sigma_{\lambda}\left(y^{\prime}\right) .
$$

Taking $g\left(y, y^{\prime}\right)=\left\|\left(y, y^{\prime}\right)-\left(z, z^{\prime}\right)\right\|^{1-\gamma}$ and applying Fatou's Lemma and Fubini's Theorem, we get

$$
\begin{array}{r}
\mathcal{J} \leq \liminf _{r \downarrow 0}(2 r)^{-1} \int_{I} \int_{\mathbf{R}} \int_{l_{t}} \int_{\left\{\left(y, y^{\prime}\right):\left|y^{\prime}-y-t\right| \leq r\right\}}\left\|\left(y, y^{\prime}\right)-\left(z, z^{\prime}\right)\right\|^{1-\gamma} d \eta(y) d \sigma_{\lambda}\left(y^{\prime}\right) \\
\times d \theta_{t, \lambda}\left(z, z^{\prime}\right) d t d \lambda \\
=\liminf _{r \downarrow 0}(2 r)^{-1} \int_{I} \iint_{K \times \mathcal{C}_{\lambda}} \int_{\left\{t:\left|y^{\prime}-y-t\right| \leq r\right\}} \int_{l_{t}}\left\|\left(y, y^{\prime}\right)-\left(z, z^{\prime}\right)\right\|^{1-\gamma} \\
\times d \theta_{t, \lambda}\left(z, z^{\prime}\right) d t d \eta(y) d \sigma_{\lambda}\left(y^{\prime}\right) d \lambda .
\end{array}
$$


To the two innermost integrals we apply the inequality (10.6) from Mattila [19]: for any Borel set $B$ and lower semicontinuous function $h$,

$$
\int_{B} \int_{l_{t}} h\left(z, z^{\prime}\right) d \theta_{t, \lambda}\left(z, z^{\prime}\right) d t \leq \iint_{\left\{\left(z, z^{\prime}\right): z^{\prime}-z \in B\right\}} h\left(z, z^{\prime}\right) d \eta(z) d \sigma_{\lambda}\left(z^{\prime}\right) .
$$

Using $h\left(z, z^{\prime}\right)=\left\|\left(y, y^{\prime}\right)-\left(z, z^{\prime}\right)\right\|^{1-\gamma}$ and $B=\left\{t:\left|y^{\prime}-y-t\right| \leq r\right\}$, we get

$$
\mathcal{J} \leq \liminf _{r \downarrow 0}(2 r)^{-1} \int_{I} \iint_{K \times \mathcal{C}_{\lambda}} \iint_{\left\{\left(z, z^{\prime}\right):\right.} \quad \begin{aligned}
& \left.\left|\left(y^{\prime}-y\right)-\left(z^{\prime}-z\right)\right| \leq r\right\} \\
& \times d \eta(z) d \sigma_{\lambda}\left(z^{\prime}\right) d \eta(y) d \sigma_{\lambda}\left(y^{\prime}\right) d \lambda .
\end{aligned}
$$

Next we change variables, and apply Fubini's Theorem:

$$
\begin{array}{r}
\mathcal{J} \leq \liminf _{r \downarrow 0}(2 r)^{-1} \iint_{K^{2}} \iint_{W^{2}} \int_{\Lambda_{r}}\left(|y-z|+\left|\Pi_{\lambda}(\omega)-\Pi_{\lambda}(\tau)\right|\right)^{1-\gamma} \\
\times d \lambda d \mu(\tau) d \mu(\omega) d \eta(y) d \eta(z) .
\end{array}
$$

where $\Lambda_{r}=\Lambda_{r}(y, z, \tau, \omega)$ was defined in (26). By Lemma 2.3(a),

$$
\mathcal{L}\left(\Lambda_{r}\right) \leq C_{1} \min \left\{1, r \lambda_{0}^{-k}\right\},
$$

and (16) implies $\left|\Pi_{\lambda}(\omega)-\Pi_{\lambda}(\tau)\right| \geq \delta_{I} \lambda_{0}^{k}$. We obtain

$$
\int_{\Lambda_{r}} \leq \frac{\mathcal{L}\left(\Lambda_{r}(y, z, \tau, \omega)\right)}{\left(|y-z|+\delta_{I} \lambda_{0}^{k}\right)^{\gamma-1}} \leq \frac{C \min \left\{1, r \lambda_{0}^{-k}\right\}}{\left(|y-z|+\lambda_{0}^{k}\right)^{\gamma-1}}
$$

Next we write

$$
\iint_{K^{2}} \iint_{W^{2}}=\iint_{\{(y, z):|y-z|<2 r\}} \iint_{W^{2}}+\iint_{\{(y, z):|y-z| \geq 2 r\}} \iint_{W^{2}}:=\mathcal{I}_{1}+\mathcal{I}_{2}
$$

and show that $\mathcal{I}_{1} \leq \mathrm{Cr}$ and $\mathcal{I}_{2} \leq \mathrm{Cr}$. The rest of the proof repeats that of Theorem 2.1 almost identically. We estimate the denominator as follows:

$$
\left(|y-z|+\lambda_{0}^{k}\right)^{\gamma-1}> \begin{cases}\lambda_{0}^{k(\gamma-1)}, & \text { if }|y-z|<2 r \\ |y-z|^{\gamma-1}, & \text { if }|y-z| \geq 2 r\end{cases}
$$

Thus, by (12), since $(\mu \times \mu)\{(\omega, \tau):|\omega \wedge \tau|=k\}<m^{-k}$,

$$
\mathcal{I}_{1} \leq C(2 r)^{\alpha} \sum_{k=N_{I}}^{\infty} \min \left\{1, r \lambda_{0}^{-k}\right\} \lambda_{0}^{k(1-\gamma)} m^{-k} .
$$

Then, splitting the sum at $k \approx \frac{\log (1 / r)}{\log \left(1 / \lambda_{0}\right)}$ as in the proof of Theorem 2.1, we get

$$
\mathcal{I}_{1} \leq C r^{\alpha}\left(\lambda_{0}^{1-\gamma} m^{-1}\right)^{\frac{\log r}{\log \lambda_{0}}}
$$

Taking logarithms and using $\alpha+\frac{\log m}{\log \left(1 / \lambda_{0}\right)}>\gamma$, we see that $\mathcal{I}_{1} \leq C r$.

To estimate $\mathcal{I}_{2}$ we observe that when $|y-z| \geq 2 r$, the set $\Lambda_{r}(y, z, \tau, \omega)$ is empty unless $\lambda_{1}^{k} \geq|y-z| / C_{2}$, where $C_{2}>0$ is from (18). Denote $\kappa(y, z)=\frac{\log \left(C_{2}^{-1}|y-z|\right)}{\log \lambda_{1}}$. 
Then

$$
\begin{aligned}
\mathcal{I}_{2} & \leq \iint_{K^{2}} \sum_{k \leq \kappa(y, z)} r \lambda_{0}^{-k}|y-z|^{1-\gamma} m^{-k} d \eta(y) d \eta(z) \\
& \leq C r \iint_{K^{2}}\left(\lambda_{0} m\right)^{-\kappa(y, z)}|y-z|^{1-\gamma} d \eta(y) d \eta(z) \\
& =C^{\prime} r \iint_{K^{2}}|y-z|^{1-\gamma-\frac{\log \left(\lambda_{0} m\right)}{\log \lambda_{1}}} d \eta(y) d \eta(z)
\end{aligned}
$$

By (31) and (12), the last integral converges, so $\mathcal{I}_{2} \leq C^{\prime \prime} r$. We have shown (33), so the proof of Theorem 1.2 is complete.

\section{Densities in $L^{q}$ : Proof of Theorem 1.3}

In this section we prove a generalization of Theorem 1.3. In order to formulate it, we need the notion of the $L^{q}$-dimension of a measure $\mu$ on the product space $\Omega$ (see e.g. Strichartz [28] for the definition of $L^{q}$ dimensions of a measure in $\mathbf{R}^{n}$ ). A cylinder set of order $k$ is a set $\left\{\omega \in \Omega: \omega_{i}=u_{i}, i=0,1, \ldots, k-1\right\}$ for some $u=u_{0} u_{1} \ldots u_{k-1}$ with $u_{i} \in\{1, \ldots, m\}$. Denote by $\mathcal{W}_{k}$ the family of all cylinder sets of order $k$. Define for $q>1$ :

$$
D_{q}(\mu)=\frac{1}{q-1} \liminf _{k \rightarrow \infty} \frac{-\log \sum_{W \in \mathcal{W}_{k}}[\mu W]^{q}}{k \log m} .
$$

The quantity $D_{2}(\mu)$ is sometimes called the correlation dimension; it can be expressed as follows:

$$
D_{2}(\mu)=\liminf _{k \rightarrow \infty} \frac{-\log \mu_{k}}{k \log m}
$$

where $\mu_{k}=(\mu \times \mu)\{(\omega, \tau):|\omega \wedge \tau| \geq k\}$.

Theorem 4.1. Let $\mu$ be a finite measure on $\Omega$ and $\nu_{\lambda}$ a family of measures on $\mathbf{R}$ defined by (14). Let $J \subset(0,1)$ be an open interval on which $\Pi_{\lambda}$ satisfies the transversality condition (11).

(a) Let $q \in(1,2]$. Then for Lebesgue-a.e. $\lambda \in J$ such that $\lambda>m^{-D_{q}(\mu)}$, the measure $\nu_{\lambda}$ is absolutely continuous with a density in $L^{q}$.

(b) For any $q>1$ and all $\lambda \in(0,1)$, if $\nu_{\lambda}$ is absolutely continuous, then

$$
\frac{d \nu_{\lambda}}{d x} \in L^{q}(\mathbf{R}) \Rightarrow \lambda \geq m^{-D_{q}(\mu)} .
$$

(c) Suppose that $\mu$ is shift-invariant and ergodic. Denote by $h(\mu)$ the entropy of $\mu$ with respect to the shift. Then the measure $\nu_{\lambda}$ is absolutely continuous for a.e. $\lambda>e^{-h(\mu)}$ such that $\lambda \in J$, and singular for all $\lambda<e^{-h(\mu)}$.

Proof. (a) Let $I=\left[\lambda_{0}, \lambda_{1}\right]$ be a subinterval of $J$, such that $\lambda_{0}>m^{-D_{q}(\mu)}$. Set $\alpha=q-1$; we have $\alpha \in(0,1]$ by assumption. We are going to prove that

$$
S:=\int_{I} \int_{\mathbf{R}}\left(\underline{D}\left(\nu_{\lambda}, x\right)\right)^{\alpha} d \nu_{\lambda}(x) d \lambda<\infty,
$$

where

$$
\underline{D}\left(\nu_{\lambda}, x\right)=\liminf _{r \downarrow 0}(2 r)^{-1} \nu_{\lambda}\left[B_{r}(x)\right]
$$


Then by Mattila [19], 2.12, the measure $\nu_{\lambda}$ is absolutely continuous for a.e. $\lambda \in I$. For such $\lambda$ we will have $\underline{D}\left(\nu_{\lambda}, x\right)=\frac{d \nu_{\lambda}}{d x}$ and $d \nu_{\lambda}(x)=\frac{d \nu_{\lambda}}{d x} d x$, so $\frac{d \nu_{\lambda}}{d x} \in L^{q}(\mathbf{R})$ for a.e. $\lambda \in I$.

First we apply Fatou's Lemma and then make a change of variable to obtain

$$
\begin{aligned}
S & \leq \liminf _{r \downarrow 0}(2 r)^{-\alpha} \int_{I} \int_{\mathbf{R}}\left(\nu_{\lambda}\left[B_{r}(x)\right]\right)^{\alpha} d \nu_{\lambda}(x) d \lambda \\
& =\liminf _{r \downarrow 0}(2 r)^{-\alpha} \int_{I} \int_{\Omega}\left(\nu_{\lambda}\left[B_{r}\left(\Pi_{\lambda}(\omega)\right)\right]\right)^{\alpha} d \mu(\omega) d \lambda .
\end{aligned}
$$

Next we reverse the order of integration and use Hölder's inequality $\int_{I} f^{\alpha} \leq$ $C\left(\int_{I} f\right)^{\alpha}$ for $\alpha \in(0,1]$ and $f \geq 0$, to get

$$
S \leq C \liminf _{r \downarrow 0}(2 r)^{-\alpha} \int_{\Omega}\left(\int_{I} \nu_{\lambda}\left[B_{r}\left(\Pi_{\lambda}(\omega)\right)\right] d \lambda\right)^{\alpha} d \mu(\omega) .
$$

We have

$$
\begin{aligned}
\int_{I} \nu_{\lambda}\left[B_{r}\left(\Pi_{\lambda}(\omega)\right)\right] d \lambda & =\int_{I} \int_{\mathbf{R}} \mathbf{1}_{B_{r}\left(\Pi_{\lambda}(\omega)\right)} d \nu_{\lambda} d \lambda \\
& =\int_{I} \int_{\Omega} \mathbf{1}_{\left\{\tau:\left|\Pi_{\lambda}(\omega)-\Pi_{\lambda}(\tau)\right| \leq r\right\}} d \mu(\tau) d \lambda \\
& =\int_{\Omega} \mathcal{L}\left(\Phi_{r}(\omega, \tau)\right) d \mu(\tau)
\end{aligned}
$$

where

$$
\Phi_{r}(\omega, \tau)=\left\{\lambda \in I:\left|\Pi_{\lambda}(\omega)-\Pi_{\lambda}(\tau)\right| \leq r\right\}
$$

Thus,

$$
S \leq C \liminf _{r \downarrow 0}(2 r)^{-\alpha} \int_{\Omega}\left(\int_{\Omega} \mathcal{L}\left(\Phi_{r}(\omega, \tau)\right) d \mu(\tau)\right)^{\alpha} d \mu(\omega) .
$$

Lemma 4.2. There exists $C>0$ such that for all $\omega$ and $\tau$ in $\Omega$,

$$
\mathcal{L}\left(\Phi_{r}(\omega, \tau)\right) \leq \operatorname{Cr} \lambda_{0}^{-|\omega \wedge \tau|} .
$$

Proof. Let $k=|\omega \wedge \tau|$. We have

$$
\phi_{\omega, \tau}(\lambda)=\Pi_{\lambda}(\omega)-\Pi_{\lambda}(\tau)=\lambda^{k} \sum_{j=0}^{\infty} b_{j}(\lambda) \lambda^{j},
$$

where $b_{j}(\lambda) \in D(\lambda)-D(\lambda)$ and $b_{0}(\lambda) \not \equiv 0$. Let $\psi(\lambda)=\sum_{j=0}^{\infty} b_{j}(\lambda) \lambda^{j}$. If we show that

$$
\mathcal{L}\{\lambda \in I:|\psi(\lambda)| \leq \rho\} \leq C \rho
$$

for $\rho>0$, the lemma will follow by taking $\rho=\lambda_{0}^{-k} r$. The class of all functions $\psi$ (corresponding to all possible choices of $b_{j}(\lambda) \in D(\lambda)-D(\lambda)$ with $\left.b_{0}(\lambda) \not \equiv 0\right)$ is compact in $C^{1}(I)$. Then it follows easily from the transversality condition (11) that for some $\epsilon>0$, depending on $I$ only,

$$
|\psi(\lambda)|<\epsilon \Rightarrow\left|\psi^{\prime}(\lambda)\right|>\epsilon .
$$

If $\rho \geq \epsilon$ then (36) holds with $C=1 / \epsilon$. Otherwise,

$$
\{\lambda \in I:|\psi(\lambda)| \leq \rho\} \subset \Psi_{\epsilon}:=\{\lambda \in I:|\psi(\lambda)| \leq \epsilon\} .
$$


The property (37) implies that $\Psi_{\epsilon}$ is a union of intervals of monotonicity for $\psi$. In each of these intervals, the portion where $|\psi(\lambda)|<\rho$ has Lebesgue measure at most $2 \rho / \epsilon$. On the other hand, $\left|\psi^{\prime}(\lambda)\right|$ has a uniform upper bound on $I$, so each of these intervals (except maybe the first and the last one) has length $\geq \epsilon / C^{\prime}$. Thus, their number is certainly less that $C^{\prime} / \epsilon$, and so $\mathcal{L}\{\lambda \in I:|\psi(\lambda)| \leq \rho\} \leq\left(C^{\prime} / \epsilon\right)(2 \rho / \epsilon)$. This proves (36).

Let us continue with the proof of part (a). By Lemma 4.2, we get from (35):

$$
S \leq C \liminf _{r \downarrow 0}(2 r)^{-\alpha} \int_{\Omega}\left(\int_{\Omega} r \lambda_{0}^{-|\omega \wedge \tau|} d \mu(\tau)\right)^{\alpha} d \mu(\omega) .
$$

Denote by $W_{\omega, k}$ the cylinder set $\left\{\tau \in \Omega: \tau_{j}=\omega_{j}, j<k\right\}$ of order $k$. Clearly, $\{\tau \in \Omega:|\omega \wedge \tau|=k\} \subset W_{\omega, k}$ for $k \geq 1$. We have

$$
S \leq C \int_{\Omega}\left(1+\sum_{k=1}^{\infty} \lambda_{0}^{-k} \mu W_{\omega, k}\right)^{\alpha} d \mu(\omega) .
$$

Now apply the inequality $\left(\sum b_{i}\right)^{\alpha} \leq \sum b_{i}^{\alpha}$ for $b_{i} \geq 0$ and $\alpha \in(0,1]$, to get

$$
\begin{aligned}
S & \leq C\left[1+\int_{\Omega} \sum_{k=1}^{\infty} \lambda_{0}^{-k \alpha}\left(\mu W_{\omega, k}\right)^{\alpha} d \mu(\omega)\right] \\
& =C_{1}+C_{2} \sum_{k=1}^{\infty} \sum_{W \in \mathcal{W}_{k}} \lambda_{0}^{-k \alpha}(\mu W)^{\alpha+1} \\
& =C_{1}+C_{2} \sum_{k=1}^{\infty} \lambda_{0}^{-k(q-1)} \sum_{W \in \mathcal{W}_{k}}(\mu W)^{q}
\end{aligned}
$$

Let $\delta>0$ be such that

$$
\lambda_{0}>m^{-\left(D_{q}(\mu)-\delta\right)}
$$

By the definition of $D_{q}(\mu)$, for $k$ sufficiently large,

$$
\sum_{W \in \mathcal{W}_{k}}(\mu W)^{q}<m^{-k\left(D_{q}(\mu)-\delta\right)(q-1)},
$$

so we obtain

$$
S \leq C_{1}+C_{2} \sum_{k=0}^{\infty}\left(\lambda_{0}^{-1} m^{-\left(D_{q}(\mu)-\delta\right)}\right)^{(q-1) k} .
$$

The series converges by (38), and the proof of part (a) is complete.

(b) Now $q \in(1, \infty)$. Suppose that $\nu_{\lambda}$ is absolutely continuous with a density $\frac{d \nu_{\lambda}}{d x}$ in $L^{q}(\mathbf{R})$. Then

$$
\lim _{r \rightarrow 0}(2 r)^{-q} \int_{\mathbf{R}}\left(\nu_{\lambda}\left[B_{r}(x)\right]\right)^{q} d x=\int_{\mathbf{R}}\left|\frac{d \nu_{\lambda}}{d x}\right|^{q} d x<\infty .
$$

This follows from the Hardy-Littlewood Maximal Theorem, or see Hardy and Littlewood [9], Th.22, for an elementary proof. We have for $k>0$ :

$$
\nu_{\lambda}=\sum_{W \in \mathcal{W}_{k}} \nu_{\lambda}^{W}, \quad \text { where } \quad \nu_{\lambda}^{W}:=\left.\mu\right|_{W} \circ \Pi_{\lambda}^{-1} .
$$


Observe that each measure $\nu_{\lambda}^{W}$ is supported on the set $\Pi_{\lambda}(W)$. Let $I_{W}$ be the smallest interval containing $\Pi_{\lambda}(W)$. It is clear that $\mathcal{L}\left(I_{W}\right)=a \lambda^{k}$ for $W \in \mathcal{W}_{k}$, where $a=\operatorname{diam} \Pi_{\lambda}(\Omega)$. Set $r=a \lambda^{k}$. Then

$$
\nu_{\lambda}^{W}\left[B_{r}(x)\right]=\nu_{\lambda}^{W}(\mathbf{R})=\mu W \quad \text { for } \quad x \in I_{W}
$$

hence

$$
\int_{\mathbf{R}}\left(\nu_{\lambda}^{W}\left[B_{r}(x)\right]\right)^{q} d x \geq a \lambda^{k}(\mu W)^{q}
$$

Using the inequality $\left(\sum b_{i}\right)^{q} \geq \sum b_{i}^{q}$ for $b_{i} \geq 0$, we obtain

$$
\begin{aligned}
(2 r)^{-q} \int_{\mathbf{R}}\left(\nu_{\lambda}\left[B_{r}(x)\right]\right)^{q} d x & \geq\left(2 a \lambda^{k}\right)^{-q} \sum_{W \in \mathcal{W}_{k}} \int_{\mathbf{R}}\left(\nu_{\lambda}^{W}\left[B_{r}(x)\right]\right)^{q} d x \\
& \geq(2 a)^{-q} \lambda^{-q k} a \lambda^{k} \sum_{W \in \mathcal{W}_{k}}(\mu W)^{q} \\
& =C\left(\lambda^{1-q}\right)^{k} \sum_{W \in \mathcal{W}_{k}}(\mu W)^{q} .
\end{aligned}
$$

This, together with (39), implies

$$
\sum_{W \in \mathcal{W}_{k}}(\mu W)^{q} \leq C^{\prime} \lambda^{(q-1) k} .
$$

Taking logarithms, dividing by $-k \log m$ and taking lim inf, yields

$$
D_{q}(\mu) \geq \log \lambda /(-\log m) .
$$

Hence $\lambda \geq m^{-D_{q}(\mu)}$, as desired.

(c) For $\omega \in \Omega$, denote by $W_{k}(\omega)$ the cylinder of order $k$ containing $\omega$. The Shannon-McMillan-Breiman Theorem asserts that

$$
\lim _{k \rightarrow \infty} \frac{1}{k} \log \left(\mu W_{k}(\omega)\right)=-h(\mu) \text { a.e. }[\mu]
$$

By Egorov's theorem, for any $\epsilon>0$ there is a subset $\Omega_{\epsilon}$ of $\Omega$ such that $\mu\left(\Omega_{\epsilon}\right)>1-\epsilon$ and the convergence in (40) is uniform on $\Omega_{\epsilon}$. Denote by $\mu_{\epsilon}$ the restriction of $\mu$ to $\Omega_{\epsilon}$. By (34), $D_{q}\left(\mu_{\epsilon}\right) \geq \frac{h(\mu)}{\log m}$ for all $q>1$, so part (a) implies that the measure $\mu_{\epsilon} \Pi_{\lambda}^{-1}$ is absolutely continuous for a.e. $\lambda \in J$ greater than $e^{-h(\mu)}$. Letting $\epsilon \rightarrow 0$ proves the absolute continuity assertion of part (c). It remains to prove the singularity assertion. Billingsley [2] used the Shannon-McMillan-Breiman Theorem to deduce that the Hausdorff dimension of the measure $\mu$ equals $h(\mu) / \log m$. Here $\operatorname{dim}_{H} \mu=\inf \left\{\operatorname{dim}_{H} X \subset \Omega: \mu(X)=1\right\}$ and the space $\Omega$ is equipped with the metric $d(\omega, \tau)=m^{-|\omega \wedge \tau|}$. The map $\Pi_{\lambda}: \Omega \rightarrow \mathbf{R}$ is Hölder:

$$
\left|\Pi_{\lambda}(\omega)-\Pi_{\lambda}(\tau)\right| \leq C \lambda^{|\omega \wedge \tau|}=C[d(\omega, \tau)]^{\alpha}, \quad \text { where } \alpha=\frac{\log (1 / \lambda)}{\log m} .
$$

Thus,

$$
\operatorname{dim}_{H} \nu_{\lambda} \leq(1 / \alpha) \operatorname{dim}_{H} \mu=h(\mu) / \log (1 / \lambda) .
$$

If $\lambda<e^{-h(\mu)}$ then $\operatorname{dim}_{H} \nu_{\lambda}<1$, so $\nu_{\lambda}$ is singular. 
Remarks. (i) In part (c), a variation of the proof shows that for a.e. $\lambda<e^{-h(\mu)}$ in the transversality interval $J$, the inequality in (41) is actually an equality.

(ii) The assumption in (c) that $\mu$ is invariant and ergodic can be relaxed; it suffices that the limiting relation (40) holds $\mu$-a.e. (and the limit there could be replaced by a limsup.)

Proof of Theorem 1.3. Recall that $\mu$ is a Bernoulli measure on $\Omega$ with weights $\left(p_{1}, \ldots, p_{m}\right)$. The entropy of $\mu$ is $h(\mu)=-\sum_{i=1}^{m} p_{i} \log p_{i}$, and direct calculation shows that

$$
m^{-D_{q}(\mu)}=\left[p_{1}^{q}+\cdots+p_{m}^{q}\right]^{1 /(q-1)}
$$

(the expression following the liminf in (34) is independent of $k$ ). Thus the theorem follows immediately from Theorem 4.1.

\section{Checking transversality, and Proof of Corollaries 1.4-1.6.}

The transversality condition (11) is used in part (a) of Theorem 4.1. It also arises in the study of self-similar sets (Pollicott and Simon [24], Solomyak [25], [26]).

Recall that $D=\left\{d_{1}(\lambda), \ldots, d_{m}(\lambda)\right\}$ is a set of digits depending on $\lambda \in(0,1)$ with $d_{j} \in C^{1}[0,1]$. Let us assume also that $d_{k}(\lambda)-d_{l}(\lambda)$ is bounded away from zero for $k \neq l$, so that

$$
b=b(D)=\sup \left\{\left|\frac{d_{i}(\lambda)-d_{j}(\lambda)}{d_{k}(\lambda)-d_{l}(\lambda)}\right|: \lambda \in[0,1], i, j, k, l \leq m, k \neq l\right\}<\infty .
$$

Setting $k=|\omega \wedge \tau|$, we can write

$$
\Pi_{\lambda}(\omega)-\Pi_{\lambda}(\tau)=\lambda^{k}\left(d_{\omega_{k}}(\lambda)-d_{\tau_{k}}(\lambda)\right)\left(1+\sum_{j=1}^{\infty} \frac{d_{\omega_{j+k}}(\lambda)-d_{\tau_{j+k}}(\lambda)}{d_{\omega_{k}}(\lambda)-d_{\tau_{k}}(\lambda)} \lambda^{j}\right) .
$$

Transversality means absence of double zeros for all functions $\Pi_{\lambda}(\omega)-\Pi_{\lambda}(\tau)$. A sufficient condition for this is the absence of double zeros for power series of the form

$$
g(x)=1+\sum_{n=1}^{\infty} g_{n} x^{n}, \quad \text { with } g_{n} \in[-b, b]
$$

Let

$$
y(b)=\inf \left\{\lambda>0: \exists g \text { of the form (43) such that } g(\lambda)=g^{\prime}(\lambda)=0\right\} .
$$

Then transversality holds on $(0, y(b))$. It turns out that $y(b)$ can be estimated rather easily, using the following extension of a lemma from Peres and Solomyak [23]. We include the brief proof for completeness.

Lemma 5.1. Let $b \geq 1$. Suppose that for some $k=k(b) \geq 1$ and $\gamma=\gamma(b) \in \mathbf{R}$ there exists a function

$$
f_{b}(x)=1-\sum_{n=1}^{k-1} b x^{n}+\gamma x^{k}+\sum_{n=k+1}^{\infty} b x^{n}
$$

such that for some $x_{b} \in(0,1)$

$$
f_{b}\left(x_{b}\right)>0 \quad \text { and } \quad f_{b}^{\prime}\left(x_{b}\right)<0 .
$$

Then $y(b)>x_{b}$. 
Proof. Since $f_{b}^{\prime \prime}$ has at most one zero on $(0,1)$, it easily follows that $f_{b}(x)>\delta$ and $f_{b}^{\prime}(x)<-\delta$ for all $x \in\left[0, x_{b}\right]$. (Consider separately the cases $k=1$ and $k>1$ ).

Let $g(x)$ be a power series of the form (43) and consider $h(x)=g(x)-f_{b}(x)$. Then (43) and the definition of $f_{b}$ imply that $h(x)=\sum_{i=1}^{l} c_{i} x^{i}-\sum_{i=l+1}^{\infty} c_{i} x^{i}$, where $c_{i} \geq 0$ and $l=k-1$ or $l=k$. Thus for any $x \in\left[0, x_{b}\right]$,

$$
g(x)<\delta \Rightarrow h(x)<0 \Rightarrow h^{\prime}(x)<0 \Rightarrow g^{\prime}(x)<-\delta,
$$

where the middle implication is a consequence of one coefficient sign change of $h$. Hence $g$ has no double zeros in $\left[0, x_{b}\right]$, and the lemma follows from the definition of $y(b)$.

Corollary 5.2. The transversality condition holds on the interval $(0, y(b))$, where $b$ is defined in (42) and

(i) $y(1)>0.64, \quad y(2)=0.5, \quad y(3)>0.415$;

(ii) $y(b) \geq(1+\sqrt{b})^{-1}$ for all $b \geq 1$ and $y(b)=(1+\sqrt{b})^{-1}$ for $b \geq 3+\sqrt{8}$.

Proof. (i) Let $f_{1}(x)=1-x-x^{2}-x^{3}+0.1 x^{4}+\sum_{n=5}^{\infty} x^{n}$. Then $f_{1}(0.64)>0$ and $f_{1}^{\prime}(0.64)<0$, so $y(1)>0.64$ by Lemma 5.1. The function $f_{2}(x)=1-2 x-2 x^{2}+$ $2 x^{3}+\sum_{n=4}^{\infty} 2 x^{n}$ satisfies $f_{2}(x)>0, f_{2}^{\prime}(x)<0$ for $x \in(0,0.5)$, and has a double zero at 0.5 ; hence $y(2)=0.5$. The function $f_{3}(x)=1-3 x-0.7 x^{2}+\sum_{n=3}^{\infty} 3 x^{n}$ satisfies $f_{3}(0.415)>0$ and $f_{3}^{\prime}(0.415)<0$; hence $y(3)>0.415$. We found the functions $f_{1}$ and $f_{3}$ using Mathematica.

(ii) The inequality $y(b) \geq(1+\sqrt{b})^{-1}$ follows from the proof of Lemma 1 in Pollicott and Simon [24]. Alternatively, let $f_{b}(x)=1-(1+2 \sqrt{b}) x+\sum_{n=2}^{\infty} b x^{n}$. It is easy to check that $f_{b}(x)>0$ and $f_{b}^{\prime}(x)<0$ for $x \in\left(0,(1+\sqrt{b})^{-1}\right)$, so Lemma 5.1 implies the desired estimate. Furthermore, $(1+\sqrt{b})^{-1}$ is a double zero for $f_{b}$; hence $y(b)=(1+\sqrt{b})^{-1}$ provided $1+2 \sqrt{b} \leq b$ (so that all coefficients are not greater than $b$ in modulus), which is equivalent to $b \geq 3+\sqrt{8}$.

In the opposite direction, it is known that the whole interval $\left(2^{-1 / 2}, 1\right)$ is filled with double zeros of power series with coefficients $-1,0,1$ (see Solomyak [26]), so the transversality condition for $m=2$ does not hold there. In Solomyak [25] it is shown that $\lambda=0.682328 \ldots$, the positive root of $x^{3}+x=1$, is also a double zero of such a series.

Proof of Corollary 1.4. To deduce the statement from Theorem 1.3, we must verify the transversality condition. Since $m=2$, we have $b(D)=1$ in (42), so transversality holds on $(0,0.64)$ by Corollary $5.2(\mathrm{i})$. For $p \in[1 / 3,2 / 3]$ and $q \leq 2$ we have $\left[p^{q}+(1-p)^{q}\right]^{\frac{1}{q-1}} \leq p^{2}+(1-p)^{2} \leq(1 / 3)^{2}+(2 / 3)^{2}=5 / 9<0.64$, so it remains to show that $\nu_{\lambda}^{p}$ has a density in $L^{2}$ for a.e. $\lambda \in(0.64,1)$.

Writing $\sum \pm \lambda^{n}=\sum \pm\left(\lambda^{2}\right)^{n}+\lambda \sum \pm\left(\lambda^{2}\right)^{n}$, we see that

$$
\widehat{\nu}_{\lambda}^{p}(u)=\widehat{\nu}_{\lambda^{2}}^{p}(u) \widehat{\nu}_{\lambda^{2}}^{p}(\lambda u) .
$$

If $\nu_{\lambda^{2}}^{p}$ has an $L^{2}$-density, then by Plancherel's Theorem $\widehat{\nu}_{\lambda^{2}}^{p} \in L^{2}(\mathbf{R})$; hence $\widehat{\nu}_{\lambda}^{p} \in$ $L^{1}(\mathbf{R})$ and $\nu_{\lambda}^{p}$ has continuous density. Thus, if we show that $\nu_{\lambda}^{p}$ has an $L^{2}$-density for a.e. $\lambda \in\left[p^{2}+(1-p)^{2}, \sqrt{p^{2}+(1-p)^{2}}\right]$, the result will follow by repeating the same argument. Since $p \in[1 / 3,2 / 3]$, it suffices to cover $(0.64, \sqrt{5 / 9})$.

Consider the convolution $\nu_{\lambda}^{p} * \nu_{\lambda}^{p}$. This is a self-similar measure corresponding to the digit set $D=\{-2,0,2\}$, with probabilities $\left(p^{2}, 2 p(1-p),(1-p)^{2}\right)$. We have 
the following implications:

$$
\frac{d\left(\nu_{\lambda}^{p} * \nu_{\lambda}^{p}\right)}{d x} \in L^{2} \Rightarrow \widehat{\nu_{\lambda}^{p} * \nu_{\lambda}^{p}} \in L^{2} \Rightarrow \widehat{\nu}_{\lambda}^{p} \in L^{4} \Rightarrow \widehat{\nu}_{\sqrt{\lambda}}^{p} \in L^{2} \Rightarrow \frac{d \nu_{\sqrt{\lambda}}^{p}}{d x} \in L^{2},
$$

using (44) in the third implication. For $\nu_{\lambda}^{p} * \nu_{\lambda}^{p}$ we have $b(D)=2$, so by Corollary $5.2(\mathrm{i})$, transversality holds on $(0,1 / 2)$. By Theorem 4.1 , the convolution $\nu_{\lambda}^{p} * \nu_{\lambda}^{p}$ has an $L^{2}$-density for a.e. $\lambda \in\left(p^{4}+4 p^{2}(1-p)^{2}+(1-p)^{4}, 1 / 2\right)$. One can check that $p^{4}+4 p^{2}(1-p)^{2}+(1-p)^{4}$ is maximal when $p=1 / 3$, so this interval contains $(11 / 27,1 / 2)$. We conclude that $\nu_{\lambda}^{p}$ has an $L^{2}$-density for a.e. $\lambda \in(\sqrt{11 / 27}, 1 / \sqrt{2})$. Since $\sqrt{11 / 27}<0.64$, we have covered $(0,1 / \sqrt{2})$.

Finally, consider $\nu_{\lambda}^{p} * \nu_{\lambda}^{p} * \nu_{\lambda}^{p}$. This is a self-similar measure with the digit set $\{-3,-1,1,3\}$ and probabilities $\left(p^{3}, 3 p^{2}(1-p), 3 p(1-p)^{2},(1-p)^{3}\right)$. As above, one can argue that if $\nu_{\lambda}^{p} * \nu_{\lambda}^{p} * \nu_{\lambda}^{p}$ has $L^{2}$-density, then $\nu_{\lambda^{1 / 3}}^{p}$ has $L^{2}$-density as well. Transversality now holds on $(0, y(3)) \supset(0,0.415)$ by Corollary $5.2(\mathrm{i})$, and after a computation, we obtain that $\nu_{\lambda}^{p}$ has $L^{2}$-density for a.e. $\lambda \in\left((245 / 729)^{\frac{1}{3}}, 0.415^{\frac{1}{3}}\right)$. Since $(245 / 729)^{\frac{1}{3}}<1 / \sqrt{2}$ and $0.415^{\frac{1}{3}}>\sqrt{5 / 9}$, we are done.

Proof of Corollary 1.5. As mentioned in the introduction, for $\lambda \in(1 / 2,1)$ the density of $\eta_{\lambda}$ is continuous. To see that transversality holds for $\lambda \in(0,1 / 2)$, recall that the digit set defining $\eta_{\lambda}$ is $D=\{-1,0,1\}$, so $b=b(D)=2$ and $y(b)=1 / 2$ by Lemma 5.2(i).

Proof of Corollary 1.6. For a.e. $\lambda \in(1 / 2,1)$ we already know that $\widehat{\nu_{\lambda}^{1 / 2}}$ is in $L^{2}$, so we may restrict attention to $\lambda \in(0,1 / 2)$. The $n$th convolution power of $\nu_{\lambda}^{1 / 2}$ is a self-similar measure assigning binomial probabilities $\left(\begin{array}{l}n \\ k\end{array}\right) 2^{-n}$ for $k=0, \ldots, n$ to the $n+1$ digits $\{-n, 2-n, \ldots, n\}$. The sum of squares of these probabilities is precisely $\tilde{\lambda}_{n}=\left(\begin{array}{c}2 n \\ n\end{array}\right) 2^{-2 n}$. It follows from Theorem 1.3 that the Fourier transform of the $n$th convolution power of $\nu_{\lambda}^{1 / 2}$ is not in $L^{2}$ if $\lambda<\tilde{\lambda}_{n}$, but is in $L^{2}$ for a.e. $\lambda \in\left(\tilde{\lambda}_{n}, y(n)\right)$, where $y(n)$ is estimated in Corollary 5.2. Using Stirling's formula with remainder, or directly by induction, it is easily verified that $y(n+1)>\tilde{\lambda}_{n}$ for all $n \geq 2$. This implies that the intervals $\left(\tilde{\lambda}_{n}, y(n)\right)$ for $n \geq 2$ form a cover of $(0,1 / 2)$, and the proof is complete.

\section{Concluding Remarks and unsolved Problems}

1. The study of arithmetic differences and sums of Cantor sets has been motivated by a question of Palis and Takens whether it is true, at least generically or typically, that if the difference of two affine Cantor sets has positive Lebesgue measure, it must have non-empty interior. This problem remains open even for middle$\alpha$ Cantor sets, although some information is contained in the work of Mendes and Oliveira [20]. An important step was the formulation by Keane and Smorodinsky of a simpler problem involving a self-similar Cantor set defined by a nonstandard digit set (see Keane, Smorodinsky and Solomyak [15], Pollicott and Simon [24]).

2. If in Theorem 2.1 one could show that the convolution considered there typically has a continuous density, this would yield a positive answer to Palis and Takens' question, at least for middle- $\alpha$ Cantor sets.

3. Is the transversality condition assumed in Theorems 1.3 and 4.1 really needed? It is certainly used in our proofs, but perhaps it could be removed by a more careful study of double zeros for the relevant power series. 
4. As mentioned in the introduction, each of the exceptional sets (which have Lebesgue measure 0) of parameters $\lambda$ in Theorems 1.1-1.3, is certainly nonempty; are these sets countable?

In Theorem 1.1(a) it can be shown that the dimension of the exceptional set in certain closed subintervals is strictly less than 1 (analogously to estimates of Pollicott and Simon [24]); in the other results of this paper such dimension estimates seem harder to establish, and it would be particularly interesting to obtain such estimates in Corollary 1.4.

5. This question concerns the middle- $\alpha$ sets defined in (1). Is it true that for a.e. pair $(\gamma, \lambda)$ such that $\operatorname{dim}_{H} \mathcal{K}_{\gamma}+\operatorname{dim}_{H} \mathcal{K}_{\lambda}>1$, the inequality

$$
\operatorname{dim}_{H}\left[\left(\mathcal{K}_{\gamma}+t\right) \cap \mathcal{K}_{\lambda}\right] \leq \operatorname{dim}_{H} \mathcal{K}_{\gamma}+\operatorname{dim}_{H} \mathcal{K}_{\lambda}-1
$$

holds for all $t \in \mathbf{R}$ ?

This is analogous to a conjecture of Furstenberg [8] concerning pairs of Cantor sets in $[0,1]$ that are invariant under multiplication mod 1 by different (multiplicatively independent) integers.

\section{ACKNOWLEDGMENTS}

We are grateful to Mark Pollicott for suggesting what the critical parameter for absolute continuity in Corollary 1.4 should be, and to Jörg Schmeling for useful discussions.

\section{REFERENCES}

1. K. Baron, A. Simon and P. Volkmann (1994). Solutions d'une équation fonctionelle dans l'espace des distributions tempérées, C. R. Acad. Sci. Paris 319, Série I, 1249-1252. MR 96b:39023

2. P. Billingsley (1960). Hausdorff dimension in probability theory, Illinois J. of Math. 4, 187209. MR 24:A1750

3. J.M. Borwein and R. Girgensohn (1994). Functional equations and distribution functions, Results in Math. 26, 229-237. MR 96b:39019

4. G. Derfel and R. Schilling (1996). Spatially chaotic configurations and functional equations with rescaling, J. Phys. A: Math. Gen. 29, 4537-4547. MR 97i:39012

5. P. Erdős (1939). On a family of symmetric Bernoulli convolutions, Amer. J. Math. 61 (1939), 974-975. MR 1:52a

6. P. Erdős (1940). On the smoothness properties of Bernoulli convolutions, Amer. J. Math. 62, 180-186. MR 1:139e

7. K.J. Falconer (1990). Fractal Geometry: Mathematical foundations and applications. Wiley, New York. MR 92j:28008

8. H. Furstenberg (1970). Intersections of Cantor sets and transversality of semigroups, Problems in Analysis, a Symposium in honour of S. Bochner, R. C. Gunning (Ed.), Princeton Univ. Press, 41-59. MR 50:7040

9. G. Hardy and J. Littlewood (1928). Some properties of fractional integrals, Math. Z. 27, 565-606.

10. J. Hawkes (1975). Some algebraic properties of small sets, Q. J. Math. Oxf. 26, 195-201. MR 51:10591

11. J.E. Hutchinson (1981). Fractals and self-similarity, Indiana Univ. Math. J. 30, 713-747. MR 82b: 49026

12. J.P. Kahane (1971). Sur la distribution de certaines series aleatoires, Colloque Th. Nombres [1969, Bordeaux], Bull. Soc. Math. France, Mémoire 25, 119-122. MR 50:12946

13. R. Kaufman (1968). On Hausdorff dimension of projections, Mathematika 15, 153-155. MR 40:2030

14. M. Keane and M. Smorodinsky (1990). On the morphology of sums of Cantor sets, unpublished manuscript. 
15. M. Keane, M. Smorodinsky and B. Solomyak (1996), Morphology of $\gamma$-expansions with deleted digits, Trans. Amer. Math. Soc. 347, 955-966. MR 95h:11079

16. R. Kenyon and Y. Peres (1991). Intersecting random translates of invariant Cantor sets, Inventiones math. 104, 601-629. MR 92g:28018

17. J. M. Marstrand (1954). Some fundamental geometrical properties of plane sets of fractional dimensions, Proc. Lond. Math. Soc. 4, 257-302. MR 16:121g

18. P. Mattila (1990). Orthogonal projections, Riesz capacities and Minkowski content Indiana University Math. J. 39, 185-198. MR 91d:28018

19. P. Mattila (1995). Geometry of sets and measures in Euclidean spaces, C.U.P., Cambridge. MR 96h:28006

20. P. Mendes and F. Oliveira (1994). On the topological structure of the arithmetic sum of two Cantor sets, Nonlinearity 7, 329-343. MR 95j:58123

21. S. Newhouse (1979). The abundance of wild hyperbolic sets and non-smooth stable sets for diffeomorphisms, Publ. Math. I.H.E.S. 50, 101-151. MR 82e:58067

22. J. Palis and F. Takens (1993). Hyperbolicity and sensitive chaotic dynamics at homoclinic bifurcations, C.U.P., Cambridge. MR 94h:58129

23. Y. Peres and B. Solomyak (1996). Absolute continuity of Bernoulli convolutions, a simple proof, Math. Research Letters 3:2, 231-239. MR 97i:28006

24. M. Pollicott and K. Simon (1995). The Hausdorff dimension of $\lambda$-expansions with deleted digits, Trans. Amer. Math. Soc. 347, no. 3, 967-983. MR 95h:11080

25. B. Solomyak (1995). On the random series $\sum \pm \lambda^{i}$ (an Erdös problem), Annals of Math. 142, 611-625. MR 97d:11125

26. B. Solomyak (1998). Measure and dimension for some fractal families, Math. Proc. Cambridge Phil. Soc., to appear.

27. B. Solomyak (1997). On the measure of arithmetic sums of Cantor sets, Indagationes Math., N.S. 8, 133-141.

28. R.S. Strichartz (1993). Self-similar measures and their Fourier transforms. III, Indiana Univ. Math. J. 42, 367-411. MR 94j:42025

Department of Mathematics, Hebrew University, Jerusalem, Israel

Current address: Department of Statistics, University of California, Berkeley, California 947203860

E-mail address: peres@stat.berkeley.edu

Department of Mathematics, University of Washington, Seattle, Washington 98195

E-mail address: solomyak@math.washington.edu 